\title{
VDB Analysis for Zeolites LTA Structures
}

\author{
Julietraja Konsalraj $^{1, *(\mathbb{D})}$, Venugopal Padmanabhan ${ }^{2 \mathbb{D}}$, Chellamani Perumal ${ }^{3 \oplus \mathbb{C}}$ \\ 1 Department of Mathematics, Sri Sivasubramaniya Nadar College of Engineering, Kalavakkam - 603 110, India; \\ julietrajak@ssn.edu.in (J.K.); \\ 2 Department of Mathematics, Shiv Nadar University Chennai, Kalavakkam - 603 110, India; \\ venugopalp@snuchennai.edu.in (V.P.); \\ 3 Department of Mathematics, Sacred Heart College (Autonomous), Tirupattur - 635 601, Tirupattur Dt., India; \\ joshmani238@gmail.com (C.P.); \\ * Correspondence: julietrajak@ssn.edu.in (J.K.);
}

Scopus Author ID 57218631900

Received: 12.09.2021; Revised: 14.10.2021; Accepted: 18.10.2021; Published: 21.11.2021

\begin{abstract}
A distinct number used to characterize a molecular graph's chemical structure and significant chemical properties is a topological index. Quite a number of Vertex degree-based (VDB) indices have been introduced to investigate the structure of molecular compounds. Zeolites are a widely used family of aluminosilicates that have applications in chemistry, medicine, and commercial production due to their advantageous chemical properties. To aid in their creation, computer simulations have found millions of possible specific structures for zeolites. However, only a small percentage of these structures have been created. To aid in this discovery process, we extend the literature by computing the analytical expressions of VDB indices, VDB entropy measures, and VDB irregularity indices.
\end{abstract}

Keywords: topological descriptors; VDB entropy measures; VDB Irregularity indices; zeolites LTA structures; graph-theoretical approach.

(C) 2021 by the authors. This article is an open-access article distributed under the terms and conditions of the Creative Commons Attribution (CC BY) license (https://creativecommons.org/licenses/by/4.0/).

\section{Introduction}

Zeolites are micro-porous aluminosilicates that can be distinguished by the SiliconAluminium $(\mathrm{Si} / \mathrm{Al}$ ) ratio present in their molecular structure. Presently, there are over 200 types of zeolites with a $\mathrm{Si} / \mathrm{Al}$ ratio ranging from 1 for type A zeolites to infinity for pure silicalites, which are crystalline silica modified zeolites devoid of aluminum [1]. The current and potential applications of zeolites have been well studied and documented in the literature. The intriguing properties of these materials find a wide array of applications such as sequestration and storage of nuclear wastes, as ion exchangers, as catalysts, as separators in purification drying, environmental treatment, petroleum refining, etc. Some zeolites have found commercial applications as molecular sieves, like Sodalite (SOD), Chabazite, Linde Type A (LTA), Mordenite, ZSM-5, Stilbite, Faujasite (FAU), and Tschernichite [2].

The cavities in the zeolites can be adjusted to trap different kinds of molecules like water, carbon dioxide, and other substances. They can adsorb and desorb molecules without causing damage to the crystal structure. Hence, zeolites provide a convenient way for selectively separating certain compounds, such as water or greenhouse gases [2]. Moreover, naturally found zeolites are used in bulk mineral applications as they are very cost-effective. For these reasons, computer simulations have been used to discover millions of possible zeolite frameworks [3]. However, very few of these have been discovered naturally or manufactured 
artificially. In order to aid in the creation of new zeolite structures, describing the molecular structure of zeolites through chemical graph theory is a critical step.

Graph theory is an important branch of mathematics that has several applications in various research areas, particularly mathematical chemistry. In chemical graph theory, the chemical structures are modeled as molecular graphs [4]. A topological index (or molecular descriptor) is a graph invariant that represents the graph's structure as a distinct real number. Different types of TIs have been introduced and studied in the literature. Among these, one of the most widely prevalent TIs is the VDB indices, which are calculated using the degrees of a molecular graph. The recent research on degree-based structural descriptors is the inspiration and starting point for the research in this article. Recently, Julietraja and Venugopal computed the degree-based indices for coronoid structures [5]. Julietraja et al. analyzed the VDB indices using M-polynomial and VDB entropy measures of certain classes of benzenoid systems [6-8]. The recent development of TIs in this area can be seen in [9-25].

In this paper, the analytical expressions of VDB indices, VDB entropy measures, and VDB irregularity indices for the chemical graph of zeolites (see Figure 1) have been derived using edge-partition techniques. Figures 2(a) and (b) depict the unit cell of Zeolite LTA and Z $(2 \times 2 \times 2)$. This paper builds on the existing studies [26] of analyzing the molecular structure of zeolites.

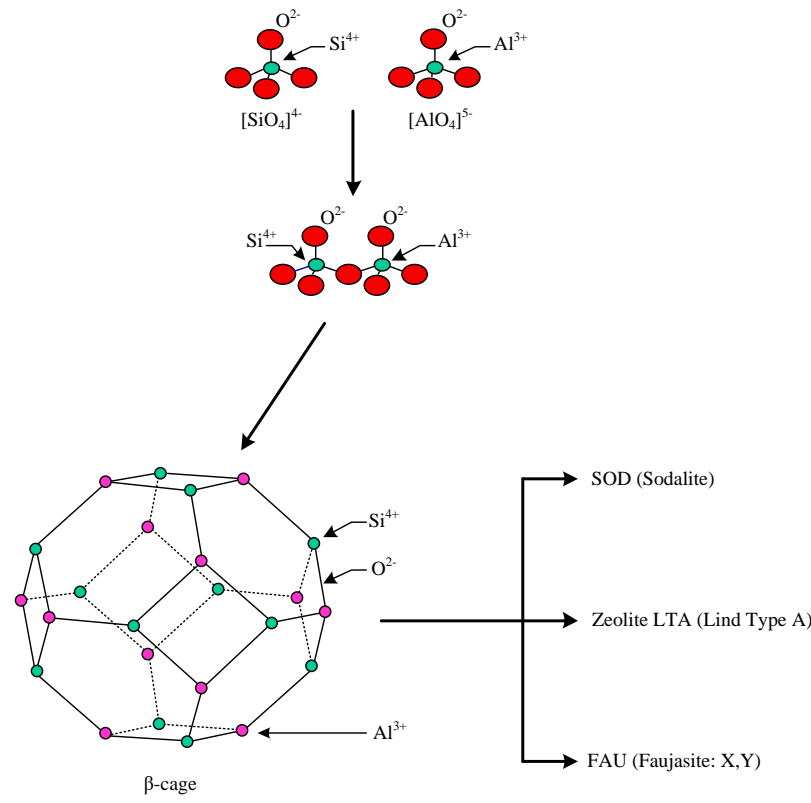

Figure 1. Construction of Zeolite LTA Structures.

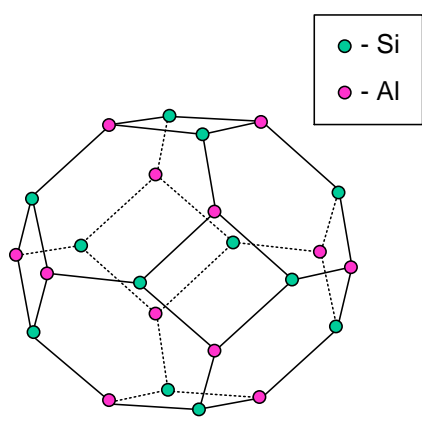

(a)

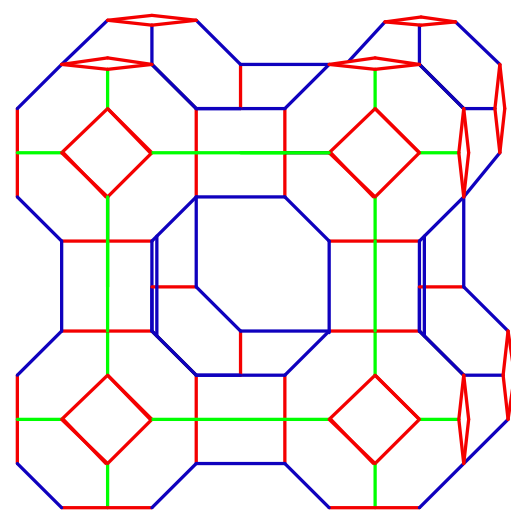

(b)

Figure 2. (a) A Unit Zeolite LTA; (b) Crystal Zeolite LTA. 


\section{Mathematical Terminologies and Literature Review}

In this section, the mathematical notations and concepts used in the article are introduced. The body of related work available in the literature on the VDB indices is also discussed briefly. In this paper, $\mathfrak{5}$ represents a connected graph, $\mathbb{V}$ and $\mathbb{E}$ depict its vertex and edge set, respectively. The degree of a vertex in a graph $\mathfrak{6} 5$ is the number of edges adjacent to that vertex. The degree of a vertex $v$ is denoted by $d_{\mathfrak{5}}(v)$ or $\operatorname{deg}_{\mathfrak{5}}(v)$ [4]. Typically, a topological index takes the following form:

$$
T I(\mathfrak{b})=\sum_{u, v \in \mathbb{V}} f(u, v)
$$

Wiener, a renowned chemist, laid the groundwork for the topological index, also known as the connectivity-based index. The Wiener index is regarded as the first topological descriptor and is widely studied among other topological indices [27]. To investigate the branching properties of alkanes, several methods are proposed based on VDB indices of the hydrogen suppressed graph $\mathfrak{6}$.

The second modified Zagreb index [28] is defined as

$$
M_{2}^{m}(\mathfrak{5})=\sum_{u v \in \mathbb{E}} \frac{1}{\left(\operatorname{deg}_{\mathfrak{5}}(u) \cdot \operatorname{deg}_{\mathfrak{5}}(v)\right)}
$$

The redefined version of Zagreb indices [29,30] are depicted as

$$
\begin{aligned}
& \operatorname{ReZG}_{1}(\mathfrak{5})=\sum_{u \in \in \mathbb{E}}\left(\frac{\operatorname{deg}_{\mathfrak{5}}(u)+\operatorname{deg}_{\mathfrak{5}}(v)}{\operatorname{deg}_{\mathfrak{5}}(u) \cdot \operatorname{deg}_{\mathfrak{5}}(v)}\right) \\
& R e Z G_{2}(\mathfrak{5})=\sum_{u \in \in \mathbb{E}} \frac{\left(\operatorname{deg}_{\mathfrak{5}}(u) \cdot \operatorname{deg}_{\mathfrak{5}}(v)\right)}{\left(\operatorname{deg}_{\mathfrak{5}}(u)+\operatorname{deg}_{\mathfrak{5}}(v)\right)} \\
& \operatorname{ReZG}(\mathfrak{b})=\sum_{u v \in \mathbb{E}}\left(\operatorname{deg}_{\mathfrak{5}}(u) \cdot \operatorname{deg}_{\mathfrak{5}}(v)\right) \cdot\left(\operatorname{deg}_{\mathfrak{5}}(u)+\operatorname{deg}_{\mathfrak{5}}(v)\right)
\end{aligned}
$$

The arithmetic-geometric index [31] is delineated as

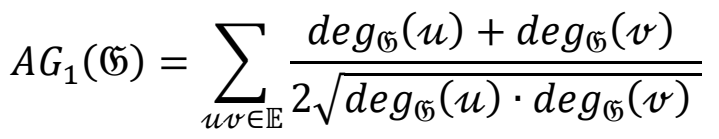

Shigehalli et al. [32] proposed the new version of VDB indices such that

$$
\begin{aligned}
S K(\mathfrak{5}) & =\sum_{u v \in \mathbb{E}}\left(\frac{\operatorname{deg}_{\mathfrak{5}}(u)+\operatorname{deg}_{\mathfrak{5}}(v)}{2}\right) \\
S K_{1}(\mathfrak{5}) & =\sum_{u \in \mathbb{E}(\mathfrak{5})}\left(\frac{\operatorname{deg}_{\mathfrak{5}}(u) \cdot \operatorname{deg}_{\mathfrak{5}}(v)}{2}\right) \\
S K_{2}(\mathfrak{5}) & =\sum_{u \in \mathbb{E}(\mathfrak{5})}\left(\frac{\operatorname{deg}_{\mathfrak{5}}(u)+\operatorname{deg}_{\mathfrak{5}}(v)}{2}\right)^{2}
\end{aligned}
$$

Furtula and Gutman [33] proposed a novel index called forgotten topological index (Findex), which is defined as

$$
F(\mathfrak{G})=\sum_{v \in \mathbb{V}} \operatorname{deg}_{\mathfrak{F}}(v)^{3}=\sum_{u v \in \mathbb{E}}\left(\operatorname{deg}_{\mathfrak{G}}(u)^{2}+\operatorname{deg}_{\mathfrak{5}}(v)^{2}\right)
$$

The symmetric division index (SDD) [34] is enacted as

$$
\operatorname{SDD}(\mathfrak{5})=\sum_{u \in \mathbb{E}}\left\{\frac{\min \left(\operatorname{deg}_{\mathfrak{5}}(u) \cdot \operatorname{deg}_{\mathfrak{5}}(v)\right)}{\max \left(\operatorname{deg}_{\mathfrak{5}}(u) \cdot \operatorname{deg}_{\mathfrak{5}}(v)\right)}+\frac{\max \left(\operatorname{deg}_{\mathfrak{5}}(u) \cdot \operatorname{deg}_{\mathfrak{5}}(v)\right)}{\min \left(\operatorname{deg}_{\mathfrak{5}}(u) \cdot \operatorname{deg}_{\mathfrak{5}}(v)\right)}\right\}
$$


The inverse sum-index [35] can provide a prediction for the total surface area of isomers. This prediction requires an elegant and simple structure that must be generated for the external graph with the help of the Math Chem. The inverse sum index of graph $\mathfrak{G}$ can be described as

$$
I(\mathfrak{5})=\sum_{u v \in \mathbb{E}}\left(\frac{\operatorname{deg}_{\mathfrak{5}}(u) \cdot \operatorname{deg}_{\mathfrak{5}}(v)}{\operatorname{deg}_{\mathfrak{5}}(u)+\operatorname{deg}_{\mathfrak{5}}(v)}\right)
$$

The sum-connectivity index [36] is described as

$$
\chi(\mathfrak{b})=\sum_{u \in \in \mathbb{E}}\left(\frac{1}{\sqrt{\operatorname{deg}_{\mathfrak{b}}(u)+\operatorname{deg}_{\mathfrak{5}}(v)}}\right)
$$

\subsection{Edge weight and degree-based entropy.}

The order of the graph is $n$ and the size is $m$ then $\varphi$ is depicted as a meaningful information function. The degree of a vertex $v$ is depicted as $\operatorname{deg}_{\mathfrak{b}}(v)$ or $d_{v}$. The Shannons entropy of a graph $(5$ is depicted [37] as

$$
\operatorname{ENT}_{\varphi}(\mathfrak{5})=-\sum_{l=1}^{n} \frac{\varphi\left(\operatorname{deg}_{\mathfrak{5}}\left(v_{l}\right)\right)}{\sum_{m=1}^{n} \varphi\left(\operatorname{deg}_{\mathfrak{5}}\left(v_{m}\right)\right)} \log \left[\frac{\varphi\left(\operatorname{deg}_{\mathfrak{5}}\left(v_{l}\right)\right.}{\sum_{m=1}^{n} \varphi\left(\operatorname{deg}_{\mathfrak{5}}\left(v_{m}\right)\right)}\right]
$$

Let $v_{l} \in \mathbb{V}(\mathfrak{G})$ and the degree of $v_{l}$ is represented by the information function $\varphi\left(v_{l}\right)$ that is, $\varphi\left(v_{l}\right)=\operatorname{deg}_{\mathfrak{5}}\left(v_{l}\right)$. Then the equation (1) can be rewritten as

$\operatorname{ENT}_{\varphi}\left(\Gamma_{1}\right)=-\sum_{l=1}^{n} \frac{\operatorname{deg}_{\mathfrak{G}}\left(v_{l}\right)}{\sum_{m=1}^{n} \operatorname{deg}_{\mathfrak{5}}\left(v_{m}\right)} \log \left[\frac{\operatorname{deg}_{\mathfrak{G}}\left(v_{l}\right)}{\sum_{m=1}^{n} \operatorname{deg}_{\mathfrak{5}}\left(v_{m}\right)}\right]$

According to the fundamental theorem of graph theory, $\sum_{m=1}^{n} \operatorname{deg}_{\mathfrak{n}}\left(v_{m}\right)=2 \mathbb{E}$. Hence, the above equation reduces to

$$
\operatorname{ENT}_{\varphi}(\mathfrak{5})=\log (2 \mathbb{E})-\frac{1}{2 \mathbb{E}} \log \left[\sum_{l=1}^{n}\left(\operatorname{deg}_{\mathfrak{5}}\left(v_{l}\right)\right)^{\operatorname{deg}_{\mathfrak{F}}\left(v_{l}\right)}\right]
$$

The entropy measure of an edge-weighted graph was first proposed by Chen et al. [38]. If $\mathfrak{G}=(\mathbb{V}(\mathfrak{b}) ; \mathbb{E}(\mathfrak{b}) ; \varphi(u v)$ is an edge-weighted graph, where $\mathbb{V}(\mathfrak{b}), \mathbb{E}(\mathfrak{b})$ and $\varphi(u v)$ represents vertex set, edge set and the edge weight of the edge $\left(l_{1} m_{1}\right)$ of $\mathfrak{G}$, then we have:

$$
\operatorname{ENT}_{\varphi}(\mathfrak{b})=-\sum_{l_{1}{ }^{\prime} m_{1}{ }^{\prime} \in \mathbb{E}} \frac{\varphi\left(u^{\prime} v^{\prime}\right)}{\sum_{w \in \mathbb{E}} \varphi(u v)} \log \left[\frac{\varphi\left(u^{\prime} v^{\prime}\right)}{\sum_{w v \in \mathbb{E}} \varphi(w v)}\right]
$$

\begin{tabular}{|c|c|c|}
\hline S.No. & Degree based entropy & Mathematical expressions \\
\hline 1. & First Zagreb entropy & $\begin{array}{l}E N T_{M_{1}}(\mathfrak{5})=\log \left(M_{1}(\mathfrak{5})\right)-\frac{1}{M_{1}(\mathfrak{5})} \log \left[\prod_{w \in \mathbb{E}}\left[\operatorname{deg}_{\mathfrak{5}}(u)+\right.\right. \\
\left.\left.\operatorname{deg}_{\mathfrak{G}}(v)\right]^{\left[\operatorname{deg}_{\mathfrak{5}}(u)+\operatorname{deg}_{\mathfrak{5}}(v)\right]}\right]\end{array}$ \\
\hline 2. & Second Zagreb entropy & $\begin{array}{l}E N T_{M_{2}}(\mathfrak{5})=\log \left(M_{2}(\mathfrak{F})\right)-\frac{1}{M_{2}(\mathfrak{F})} \log \left[\prod_{w \in \in \mathbb{E}}\left[\operatorname{deg}_{\mathfrak{5}}(u)\right.\right. \\
\left.\left.\operatorname{deg}_{\mathfrak{G}}(v)\right]^{\left[\operatorname{deg}_{\mathfrak{F}}(u) \cdot \operatorname{deg}_{\mathfrak{F}}(v)\right]}\right]\end{array}$ \\
\hline 3. & $\begin{array}{l}\text { Second modified } \\
\text { Zagreb entropy }\end{array}$ & $E N T_{M_{2}^{m}}(\mathfrak{G})=\log \left(M_{2}^{m}(\mathfrak{5})\right)-\frac{1}{M_{2}^{m}(\mathfrak{G})} \log \left[\prod_{w \in \mathbb{E}}\left[\frac{1}{\left(\operatorname{deg}_{\mathfrak{F}}(u) \cdot \operatorname{deg}_{\mathfrak{F}}(v)\right)}\right]\left[\frac{1}{\left(\operatorname{deg}_{\mathfrak{F}}(u) \cdot \operatorname{deg}_{\mathfrak{G}}(v)\right)}\right]\right.$ \\
\hline 4. & Hyper Zagreb entropy & $\begin{array}{l}\operatorname{ENT}_{H M}(\mathfrak{5})=\log (H M(\mathfrak{5}))-\frac{1}{H M(\mathfrak{5})} \log \left[\prod_{w \in \mathbb{E}}\left[\operatorname{deg}_{\mathfrak{5}}(u)+\right.\right. \\
\left.\left.\operatorname{deg}_{\mathfrak{G}}(v)\right]^{2}\left[\operatorname{deg}_{\mathfrak{F}}(u)+\operatorname{deg}_{\mathfrak{F}}(v)\right]^{2}\right]\end{array}$ \\
\hline 5. & $\begin{array}{l}\text { Augmented Zagreb } \\
\text { entropy }\end{array}$ & $E N T_{A}(\mathfrak{5})=\log (A(\mathfrak{5}))-\frac{1}{A(\mathfrak{G})} \log \left[\prod_{w \in \mathbb{E}}\left[\frac{\operatorname{deg}_{\mathfrak{5}}(u) \cdot \operatorname{deg}_{\mathfrak{5}}(v)}{\operatorname{deg}_{\mathfrak{5}}(u)+\operatorname{deg}_{\mathfrak{F}}(v)-2}\right]^{3\left[\frac{\operatorname{deg}_{\mathfrak{G}}(u) \cdot \operatorname{deg}_{\mathfrak{G}}(v)}{\operatorname{deg}_{\mathfrak{G}}(u)+\operatorname{deg}_{\mathfrak{5}}(v)-2}\right]^{3}}\right]$ \\
\hline 6. & $\begin{array}{l}\text { Atom bond connectivity } \\
\text { entropy }\end{array}$ & 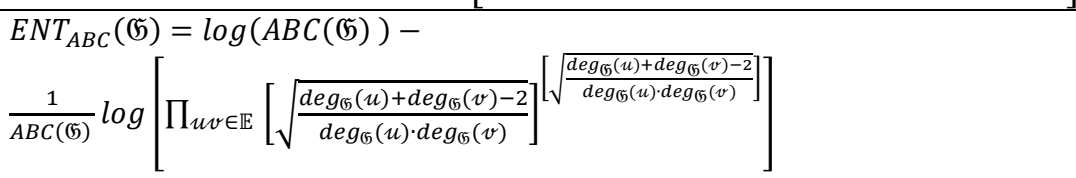 \\
\hline
\end{tabular}

By using the equations of (1), (2), and (3) we get the following entropy measures:

Table 1. Edge weight and degree-based entropy. 


\begin{tabular}{|c|c|c|}
\hline S.No. & Degree based entropy & Mathematical expressions \\
\hline 7. & $\begin{array}{l}\text { Geometric arithmetic } \\
\text { entropy }\end{array}$ & 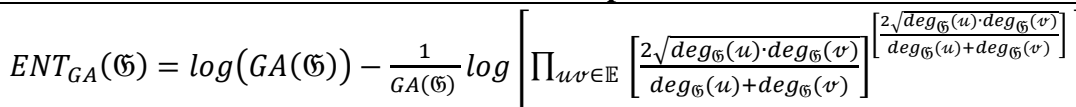 \\
\hline 8. & Forgotten entropy & $\begin{array}{l}\operatorname{ENT}_{F}(\mathfrak{5})=\log (F(\mathfrak{5}))-\frac{1}{F(\mathfrak{W})} \log \left[\prod_{w \in \mathbb{E}}\left[\operatorname{deg}_{\mathfrak{5}}(u)^{2}+\right.\right. \\
\left.\left.\operatorname{deg}_{\mathfrak{5}}(v)^{2}\right]^{\left[\operatorname{deg}_{\mathfrak{F}}(u)^{2}+\operatorname{deg}_{\mathfrak{F}}(v)^{2}\right]}\right]\end{array}$ \\
\hline 9. & $\begin{array}{l}\text { Sum-connectivity } \\
\text { entropy }\end{array}$ & $E N T_{\chi}(\mathfrak{5})=\log (\chi(\mathfrak{5}))-\frac{1}{\chi(\mathfrak{F})} \log \left[\prod_{w \in \mathbb{E}}\left[\frac{1}{\sqrt{\operatorname{deg}_{\mathfrak{F}}(u)+\operatorname{deg}_{\mathfrak{F}}(v)}}\right]^{\left[\frac{1}{\sqrt{\operatorname{deg}_{\mathfrak{5}}(u)+\operatorname{deg}_{\mathfrak{F}}(v)}}\right]}\right]$ \\
\hline 10. & $\begin{array}{l}\text { First redefined Zagreb } \\
\text { entropy }\end{array}$ & 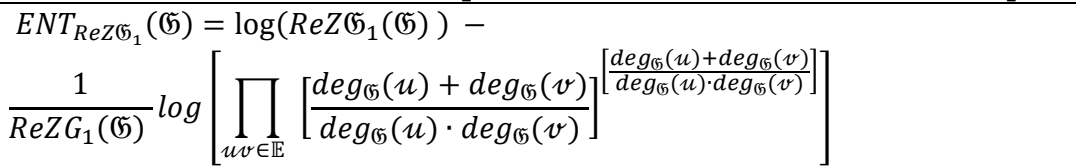 \\
\hline 11. & $\begin{array}{l}\text { Second redefined } \\
\text { Zagreb entropy }\end{array}$ & $\begin{array}{l}\operatorname{ENT}_{\operatorname{ReZG}_{2}}(\mathfrak{5})=\log \left(\operatorname{ReZ} G_{2}(\mathfrak{5})\right)- \\
\frac{1}{\operatorname{ReZG}_{2}(\mathfrak{5})} \log \left[\prod_{w \in \mathbb{E}}\left[\frac{\operatorname{deg}_{\mathfrak{G}}(u) \cdot \operatorname{deg}_{\mathfrak{G}}(v)}{\operatorname{deg}_{\mathfrak{G}}(u)+\operatorname{deg}_{\mathfrak{G}}(v)}\right]^{\left[\frac{\operatorname{deg}_{\mathfrak{G}}(u) \cdot \operatorname{deg}_{\mathfrak{G}}(v)}{\operatorname{deg}_{\mathfrak{G}}(u)+\operatorname{deg}_{\mathfrak{G}}(v)}\right]}\right]\end{array}$ \\
\hline 12. & $\begin{array}{l}\text { Third redefined Zagreb } \\
\text { entropy }\end{array}$ & $\begin{array}{l}\operatorname{ENT}_{R e Z G_{3}}(\Gamma)=\log \left(\operatorname{ReZG_{3}}(\Gamma)\right)-\frac{1}{\operatorname{ReZG}_{3}(\Gamma)} \log \left[\prod_{w \in E(\Gamma)}\left[\left(\operatorname{deg}_{\mathfrak{5}}(u) \cdot \operatorname{deg} g_{\mathfrak{5}}(v)\right) \cdot\right.\right. \\
\left.\left.\left(\operatorname{deg}_{\mathfrak{5}}(u)+\operatorname{deg}_{\mathfrak{5}}(v)\right)\right]^{\left[\left(\operatorname{deg}_{\mathfrak{5}}(u) \cdot \operatorname{deg}_{\mathfrak{5}}(v)\right) \cdot\left(\operatorname{deg}_{\mathfrak{5}}(u)+\operatorname{deg}_{\mathfrak{5}}(v)\right)\right]}\right]\end{array}$ \\
\hline
\end{tabular}

\subsection{Irregularity Indices for QSPR Studies.}

Irregularity indices are numerical parameters that quantify the irregularity of a molecular graph. The study of irregular graphs was brought to the limelight by Paul Erdös in [39]. If a topological descriptor becomes zero for a normal graph and non-zero for a non-regular graph, it is known as an irregularity index. It has been proved that using regression analysis in [40].

Table 2. Irregularity based indices for QSPR Analysis

\begin{tabular}{|c|c|}
\hline S.No. & Irregularity based indices \\
\hline 1. & $\operatorname{VAR}(\mathfrak{G})=\sum_{u \in \mathbb{V}}\left(\operatorname{deg}_{\mathfrak{F}}(u)-\frac{2 m}{n}\right)^{2}=\frac{M_{1}(\mathfrak{G})}{n}-\left(\frac{2 m}{n}\right)^{2}$ \\
\hline 2. & $I R 1(\mathfrak{F})=\sum_{u \in \mathbb{V}} \operatorname{deg}_{\mathfrak{F}}(u)^{3}-\frac{2 m}{n} \sum_{\mathfrak{u} \in \mathbb{V}} \operatorname{deg}_{\mathfrak{F}}(u)^{2}=F(\mathfrak{F})-\frac{2 m}{n} M_{1}(\mathfrak{G})$ \\
\hline 3. & $\operatorname{IR} 2(\mathfrak{5})=\sqrt{\frac{\sum_{w \in \mathbb{E}}\left(\operatorname{deg}_{\mathfrak{G}}(u) \cdot \operatorname{deg}_{\mathfrak{G}}\left(m_{1}\right)\right)}{m}}-\frac{2 m}{n}=\sqrt{\frac{M_{2}(\mathfrak{G})}{m}}-\frac{2 m}{n}$ \\
\hline 4. & 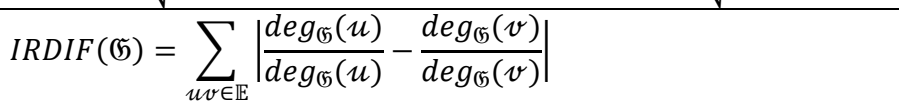 \\
\hline 5. & $A L(\mathfrak{5})=\sum_{u v \in \mathbb{E}}\left|\operatorname{deg}_{\mathfrak{F}}(u)-\operatorname{deg}_{\mathfrak{F}}(v)\right|$ \\
\hline 6. & $\operatorname{IRL}(\mathfrak{G})=\sum_{\mathfrak{w} \in \mathbb{E}}\left|\ln \operatorname{deg}_{\mathfrak{5}}(u)-\ln \operatorname{deg}_{\mathfrak{5}}(v)\right|$ \\
\hline 7. & $\operatorname{IRLU}(\mathfrak{5})=\sum_{u \in \mathbb{E}} \frac{\left|\operatorname{deg}_{\mathfrak{5}}(u)-\operatorname{deg}_{\mathfrak{5}}(v)\right|}{\min \left(\operatorname{deg}_{\mathfrak{5}}(u) \cdot \operatorname{deg}_{\mathfrak{5}}(v)\right)}$ \\
\hline 8. & $\operatorname{IRLF}(\mathfrak{5})=\sum_{u v \in \mathbb{E}} \frac{\left|\operatorname{deg}_{\mathfrak{5}}(u)-\operatorname{deg}_{\mathfrak{5}}(v)\right|}{\sqrt{\left(\operatorname{deg}_{\mathfrak{F}}(u) \cdot \operatorname{deg}_{\mathfrak{5}}(v)\right)}}$ \\
\hline 9. & $\operatorname{IRF}(\mathfrak{G})=\sum_{w \in \mathbb{E}}\left(d_{u}-d_{v}\right)^{2}=F(\mathfrak{b})-2 M_{2}(\mathfrak{G})$ \\
\hline 10. & 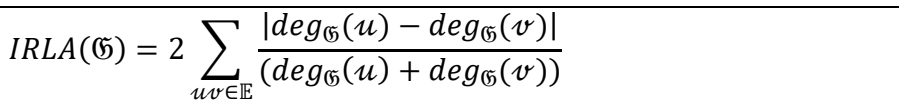 \\
\hline 11. & $\operatorname{IRD} D_{1}(\mathfrak{G})=\sum_{w \in \mathbb{E}} \ln \left\{1+\left|\operatorname{deg}_{\mathfrak{F}}(u)-\operatorname{deg}_{\mathfrak{G}}(v)\right|\right\}$ \\
\hline 12. & $\operatorname{IRA}(\mathfrak{5})=\sum_{w \in \mathbb{E}}\left(\operatorname{deg}_{\mathfrak{5}}(u)^{-1 / 2}-\operatorname{deg}_{\mathfrak{5}}(v)^{-1 / 2}\right)^{2}$ \\
\hline
\end{tabular}




\begin{tabular}{|c|c|}
\hline S.No. & Irregularity based indices \\
\hline 13. & $\operatorname{IRB}(\mathfrak{G})=\sum_{u \in \mathbb{E}}\left(\operatorname{deg}_{\mathfrak{L}}(u)^{1 / 2}-\operatorname{deg}_{\mathfrak{5}}(v)^{1 / 2}\right)^{2}$ \\
\hline 14. & $\operatorname{IRB}(\mathfrak{G})=\frac{\sum_{w \in \mathbb{E}} \sqrt{\left(\operatorname{deg}_{\mathfrak{5}}(u) \cdot \operatorname{deg}_{\mathfrak{5}}(v)\right)}}{m}-\frac{2 m}{n}=\frac{R R(\mathfrak{b})}{m}-\frac{2 m}{n}$ \\
\hline 15. & 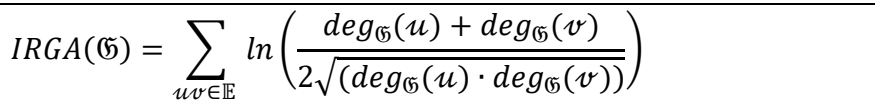 \\
\hline 16. & $I R R_{t}(\mathfrak{G})=\frac{1}{2} \sum_{w \in \mathbb{E}}\left|\operatorname{deg}_{\mathfrak{G}}(u)-\operatorname{deg}_{\mathfrak{G}}(v)\right|$ \\
\hline
\end{tabular}

\section{Results and Discussions}

This section has computed VDB indices, VDB entropy measures, and VDB irregularity indices for zeolite LTA frameworks using edge-partition techniques.

Table 3. The edge partition table of $Z[f, k, t]$.

$\left(\operatorname{deg}_{\mathfrak{G}}(u), \operatorname{deg}_{\mathfrak{F}}(v)\right)$

\begin{tabular}{c|c}
$(3,3)$ & $8(f k+k t+f t)+4(f+k+t)$ \\
\hline$(3,4)$ & $8(f k+k t+f t)+8(f+k+t)-48$ \\
\hline$(4,4)$ & $48 f k t-20(f k+k t+f t)-12(f+k+t)+48$
\end{tabular}

Theorem 1. If $Z[f, k, t]$ is a zeolite LTA framework where $f \leq k \leq t$, then VDB indices are computed as

1. $M_{2}^{m}(Z[f, k, t])=(108 f k t+11(f k+f t+k t)+13(f+k+t)-36) /$ 36

2. $\operatorname{ReZG}_{1}(Z[f, k, t])=(72 k k t+4(f+k+t)-12) / 3$

3. $R e Z G_{2}(Z[f, k, t])=(672 f k t-100(f k+f t+k t)-30(f+k+t)+$ 96) $/ 7$

4. $R e Z G_{3}(Z[f, k, t])=6144 f k t-1456(f k+f t+k t)-648(f+k+$ t) +2112

5. $S K(Z[f, k, t])=192 f k t-28(f k+f t+k t)-8(f+k+t)+24$

6. $S K_{1}(Z[f, k, t])=384 f k t-76(f k+f t+k t)-30(f+k+t)+96$

7. $S K_{2}(Z[f, k, t])=768 f k t-150(f k+f t+k t)-58(f+k+t)+180$

8. $A G_{1}(Z[f, k, t])=(144 f k t-36((f k+f t+k t)-24(f+k+t)+$ $144) / 3$

9. $F(Z[f, k, t])=1536 f k t-296(f k+f t+k t)-112(f+k+t)+336$

10. $\chi(Z[f, k, t])=\frac{1}{21}[((28 k+28 t+14) f+(28 t+14) k+14 t) \sqrt{3}+$ $((252 t-105) k-105 t-63) f+(-105 t-63) k-63 t+252)] \sqrt{2}+$ $\left(\frac{8}{7}\right) \sqrt{7}((k+t+1) f+(t+1) k+t-6)$

11. $S D D(Z[f, k, t])=(288 f k t-22(f k+f t+k t)+2(f+k+t)-12) /$ 3

Proof: Let $\mathfrak{b}=Z[f, k, t]$ be a Zeolite graph. The cardinality of $\mathfrak{G}$ is depicted as $|\mathbb{V}(\mathfrak{G})|=24 k k t$ and $|E(\mathfrak{G})|=48 k k t-4(\mathfrak{k}+k t+f t)$. Using the definitions of VDB indices and Table 3, the following results are obtained as

1. $M_{2}^{m}(Z[f, k, t])=(108 f k t+11(f k+f t+k t)+13(f+k+t)-36) /$ 36

2. $R e Z G_{1}(Z[f, k, t])=(72 k k t+4(f+k+t)-12) / 3$ 
3. $R e Z G_{2}(Z[f, k, t])=(672 f k t-100(f k+f t+k t)-30(f+k+t)+$ 96) $/ 7$

4. $R e Z G_{3}(Z[f, k, t])=6144 f k t-1456(f k+f t+k t)-648(f+k+$ t) +2112

5. $S K(Z[f, k, t])=192 f k t-28(f k+f t+k t)-8(f+k+t)+24$

6. $S K_{1}(Z[f, k, t])=384 f k t-76(f k+f t+k t)-30(f+k+t)+96$

7. $S K_{2}(Z[f, k, t])=768 f k t-150(f k+f t+k t)-58(f+k+t)+180$

8. $A G_{1}(Z[f, k, t])=(144 f k t-36((f k+f t+k t)-24(f+k+t)+$ 144)/3

9. $F(Z[f, k, t])=1536 f k t-296(f k+f t+k t)-112(f+k+t)+336$

10. $\chi(Z[f, k, t])=\frac{1}{21}[((28 k+28 t+14) f+(28 t+14) k+14 t) \sqrt{3}+$

$$
\begin{aligned}
& ((252 t-105) k-105 t-63) f+(-105 t-63) k-63 t+252)] \sqrt{2}+ \\
& \left(\frac{8}{7}\right) \sqrt{7}((k+t+1) f+(t+1) k+t-6)
\end{aligned}
$$

11. $S D D(Z[f, k, t])=(288 f k t-22(f k+f t+k t)+2(f+k+t)-12) /$ 3

Hence the proof.

\section{Computing the VDB Entropy Measures for $Z[f, k, t]$}

In this article, we compute the results for zeolite LTA frameworks. Let $\mathfrak{G}=\mathbb{Z}[\mathfrak{f}, k, t]$ be a Zeolite graph. The total number of vertices and edges of $\mathbb{G}$ is depicted as $|\mathbb{V}(\mathfrak{G})|=$

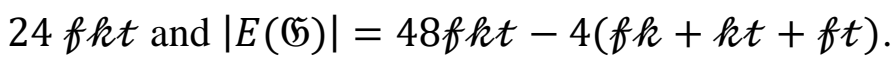

\section{First Zagreb entropy}

$M_{1}(\mathfrak{5})=384 f k t-56(f k+k t+f t)-16(f+k+t)+48$

$E N T_{M_{1}}(\mathfrak{5})=\log \left(M_{1}(\mathfrak{5})\right)-\frac{1}{M_{1}(\mathfrak{F})} \log \left[\prod_{w \in \in \mathbb{E}}\left[\operatorname{deg}_{\mathfrak{G}}(u)+\operatorname{deg}_{\mathfrak{5}}(v)\right]^{\left[\operatorname{deg}_{\mathfrak{F}}(u)+\operatorname{deg}_{\mathfrak{F}}(v)\right]}\right]$

$E N T_{M_{1}}(\mathfrak{G})=\log \left(M_{1}(\mathfrak{b})\right)-\frac{1}{M_{1}(\mathfrak{F})} \log \left[\prod_{w \in \mathbb{E}_{\{3,3\}}}\left[\operatorname{deg}_{\mathfrak{5}}(u)+\right.\right.$

$\left.\operatorname{deg}_{\mathfrak{5}}(v)\right]^{\left[\operatorname{deg}_{\mathfrak{F}}(u)+\operatorname{deg}_{\mathfrak{5}}(v)\right]} \times \prod_{u v \in \mathbb{E}_{\{3,4\}}}\left[\operatorname{deg}_{\mathfrak{5}}(u)+\operatorname{deg}_{\mathfrak{5}}(v)\right]^{\left[\operatorname{deg}_{\mathfrak{F}}(u)+\operatorname{deg}_{\mathfrak{5}}(v)\right]} \times$

$\left.\prod_{u v \in \mathbb{E}_{\{4,4\}}}\left[\operatorname{deg}_{\mathfrak{5}}(u)+\operatorname{deg}_{\mathfrak{5}}(v)\right]^{\left[\operatorname{deg}_{\mathfrak{F}}(u)+\operatorname{deg}_{\mathfrak{5}}(v)\right]}\right]$

$=\log \left(M_{1}(\mathfrak{5})\right)-\frac{1}{M_{1}(\mathfrak{G})} \log \left[\left[[8(f k+k t+f t)+4(f+k+t)] \times(6)^{6}\right] \times[[8(f k+\right.$ $\left.k t+f t)+8(f+k+t)-48] \times(7)^{7}\right] \times[[48 k k t-20(f k+k t+f t)-12(f+k+$ $\left.\left.t)+48] \times(8)^{8}\right]\right]$

Where $M_{1}(\Gamma)=384 f k t-56(f k+f t+k t)-16(f+k+t)+48$

\section{Second Zagreb entropy}

$M_{2}(\Gamma)=768 f k t-152(f k+f t+k t)-60(f+k+t)+192$

$E N T_{M_{2}}(\mathfrak{5})=\log \left(M_{2}(\mathfrak{5})\right)-\frac{1}{M_{2}(\mathfrak{G})} \log \left[\prod_{u v \in \mathbb{E}}\left[\operatorname{deg}_{\mathfrak{5}}(u) \cdot \operatorname{deg}_{\mathfrak{5}}(v)\right]^{\left[\operatorname{deg}_{\mathfrak{F}}(u) \cdot \operatorname{deg}_{\mathfrak{F}}(v)\right]}\right]$ 
$\operatorname{ENT}_{M_{2}}(\mathfrak{5})=\log \left(M_{2}(\mathfrak{b})\right)-\frac{1}{M_{2}(\mathfrak{G})} \log \left[\prod_{u v \in E_{\{3,3\}}}\left[\operatorname{deg}_{\mathfrak{5}}(u) \cdot \operatorname{deg}_{\mathfrak{5}}(v)\right]^{\left[\operatorname{deg}_{\mathfrak{F}}(u) \cdot \operatorname{deg}_{\mathfrak{F}}(v)\right]} \times\right.$ $\prod_{u v \in E_{\{3,4\}}}\left[\operatorname{deg}_{\mathfrak{5}}(u) \cdot \operatorname{deg}_{\mathfrak{5}}(v)\right]^{\left[\operatorname{deg}_{\mathfrak{5}}(u) \cdot \operatorname{deg}_{\mathfrak{5}}(v)\right]} \times \prod_{u v \in E_{\{4,4\}}}\left[\operatorname{deg}_{\mathfrak{5}}(u) \cdot\right.$

$\left.\left.\operatorname{deg}_{\mathfrak{5}}(v)\right]^{\left[\operatorname{deg}_{\mathfrak{F}}(u) \cdot \operatorname{deg}_{\mathfrak{F}}(v)\right]}\right]$

$=\log \left(M_{2}(\mathfrak{G})\right)-\frac{1}{M_{2}(\mathbb{G})} \log \left[\left[[8(f k+k t+f t)+4(f+k+t)] \times(9)^{9}\right] \times[[8(f k+\right.$ $\left.k t+f t)+8(f+k+t)-48] \times(12)^{12}\right] \times[[48 f k t-20(f k+k t+f t)-12(f+$ $\left.\left.k+t)+48] \times(16)^{16}\right]\right]$

Where $M_{2}(\mathfrak{5})=768 f k t-152(f k+f t+k t)-60(f+k+t)+192$

\section{Second modified Zagreb entropy}

$M_{2}^{m}(\mathfrak{5})=768 k k t-152(f k+f t+k t)-60(f+k+t)+192$

$E N T_{M_{2}^{m}}(\mathfrak{G})=\log \left(M_{2}^{m}(\mathfrak{b})\right)-\frac{1}{M_{2}^{m}(\mathfrak{5})} \log \left[\prod_{u v \in \mathbb{E}}\left[\frac{1}{\left(\operatorname{deg}_{\mathfrak{F}}(u) \cdot \operatorname{deg}_{\mathfrak{5}}(v)\right)}\right]^{\left[\frac{1}{\left(\operatorname{deg}_{\mathfrak{5}}(u) \cdot \operatorname{deg}_{\mathfrak{G}}(v)\right)}\right]}\right]$ $E N T_{M_{2}}(\mathfrak{5})=\log \left(M_{2}(\mathfrak{5})\right)-\frac{1}{M_{2}(\mathfrak{G})} \log \left[\prod_{w \in E_{\{3,3\}}}\left[\frac{1}{\left(\operatorname{deg}_{\mathfrak{5}}(u) \cdot \operatorname{deg}_{\mathfrak{F}}(v)\right)}\right]^{\left[\frac{1}{\left(\operatorname{leg}_{\mathfrak{5}}(u) \cdot \operatorname{deg}_{\mathfrak{5}(v))}\right.}\right]} \times\right.$ $\prod_{u v \in E_{\{3,4\}}}\left[\frac{1}{\left(\operatorname{deg}_{\mathfrak{F}}(u) \cdot \operatorname{deg}_{\mathfrak{G}}(v)\right)}\right]^{\left[\frac{1}{\left(\operatorname{deg}_{\mathfrak{G}}(u) \cdot \operatorname{deg}_{\mathfrak{F}}(v)\right)}\right]} \times$

$\left.\prod_{w \in E_{\{4,4\}}}\left[\frac{1}{\left(\operatorname{deg}_{\mathfrak{F}}(u) \cdot \operatorname{deg}_{\mathfrak{5}}(v)\right)}\right]^{\left[\frac{1}{\left(\operatorname{deg}_{\mathfrak{F}}(u) \cdot \operatorname{deg}_{\mathfrak{G}}(v)\right)}\right]}\right]$

Where $M_{2}^{m}(\mathfrak{5})=768 f k t-152(f k+f t+k t)-60(f+k+t)+192$

\section{The hyper Zagreb entropy}

$H M(\mathfrak{5})=((3072 t-600) k-600 t-232) f+(-600 t-232) k-232 t+720$

$E N T_{H M}(\mathfrak{b})=\log (H M(\mathfrak{b}))-\frac{1}{H M(\mathfrak{b})} \log \left[\prod_{u v \in \mathbb{E}}\left[\operatorname{deg}_{\mathfrak{5}}(u)+\right.\right.$ $\left.\left.\operatorname{deg}_{\mathfrak{5}}(v)\right]^{2\left[\operatorname{deg}_{\mathfrak{F}}(u)+\operatorname{deg}_{\mathfrak{F}}(v)\right]^{2}}\right]$

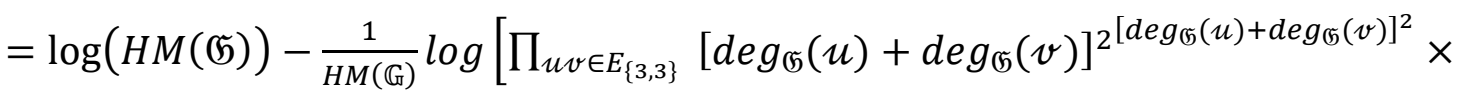

$\prod_{w \in E_{\{3,4\}}}\left[\operatorname{deg}_{\mathfrak{5}}(u)+\operatorname{deg}_{\mathfrak{5}}(v)\right]^{2\left[\operatorname{deg}_{\mathfrak{5}}(u)+\operatorname{deg}_{\mathfrak{F}}(v)\right]^{2}} \times \prod_{w \in E_{\{4,4\}}}\left[\operatorname{deg}_{\mathfrak{5}}(u)+\right.$ $\left.\operatorname{deg}_{\mathfrak{G}(v)]^{2}\left[\operatorname{deg}_{\mathfrak{5}}(u)+\operatorname{deg}_{\mathfrak{5}}(v)\right]^{2}}\right]$

$=\log (H M(\mathfrak{5}))-\frac{1}{H M(\mathfrak{5})} \log \left[\left[[8(f k+k t+f t)+4(f+k+t)] \times(36)^{36}\right] \times[[8(f k+\right.$ $\left.k t+f t)+8(f+k+t)-48] \times(49)^{49}\right] \times[[48 f k t-20(f k+k t+f t)-12(f+$ $\left.\left.k+t)+48] \times(64)^{64}\right]\right]$

Where $H M(\mathfrak{5})=((3072 t-600) k-600 t-232) f+(-600 t-232) k-232 t+720$

\section{The augmented Zagreb entropy}

$A(\Gamma)=\sum_{u \in E(\Gamma)}\left[\frac{d_{u} \cdot d_{v}}{d_{u}+d_{v}-2}\right]^{3}$ 
$\operatorname{ENT}_{A}(\mathfrak{5})=\log (A(\mathfrak{5}))-\frac{1}{A(\mathfrak{5})} \log \left[\prod_{u v \in \mathbb{E}}\left[\frac{\operatorname{deg}_{\mathfrak{5}}(u) \cdot \operatorname{deg}_{\mathfrak{5}}(v)}{\operatorname{deg}_{\mathfrak{5}}(u)+\operatorname{deg}_{\mathfrak{5}}(v)-2}\right]^{3\left[\frac{\operatorname{deg}_{\mathfrak{5}}(u) \cdot \operatorname{deg} g_{\mathfrak{5}}(v)}{\operatorname{deg}_{\mathfrak{5}}(u)+\operatorname{deg}_{\mathfrak{5}}(v)-2}\right]^{3}}\right]^{3}$ $=\log (A(\mathfrak{5}))-\frac{1}{A(\mathfrak{F})} \log \left[\prod_{w \in \in E_{\{3,3\}}}\left[\frac{\operatorname{deg}_{\mathfrak{F}}(u) \cdot \operatorname{deg}_{\mathfrak{F}}(v)}{\operatorname{deg}_{\mathfrak{F}}(u)+\operatorname{deg}_{\mathfrak{F}}(v)-2}\right]^{3\left[\frac{\operatorname{deg}_{\mathfrak{F}}(u) \cdot \operatorname{deg}_{\mathfrak{5}}(v)}{\operatorname{deg}_{\mathfrak{F}}(u)+\operatorname{deg}_{\mathfrak{F}}(v)-2}\right]^{3}} \times\right.$ $\prod_{w \in E_{\{3,4\}}}\left[\frac{\operatorname{deg}_{\mathfrak{F}}(u) \cdot \operatorname{deg}_{\mathfrak{F}}(v)}{\operatorname{deg}_{\mathfrak{F}}(u)+\operatorname{deg}_{\mathfrak{F}}(v)-2}\right]^{3\left[\frac{\operatorname{deg}_{\mathfrak{G}}(u) \cdot \operatorname{deg} g_{\mathfrak{5}}(v)}{\operatorname{deg}_{\mathfrak{F}}(u)+\operatorname{deg}_{\mathfrak{F}}(v)-2}\right]^{3}} \times$ $\left.\prod_{u v \in E_{\{4,4\}}}\left[\frac{\operatorname{deg}_{\mathfrak{5}}(u) \cdot \operatorname{deg}_{\mathfrak{5}}(v)}{\operatorname{deg}_{\mathfrak{5}}(u)+\operatorname{deg}_{\mathfrak{5}}(v)-2}\right]^{3\left[\frac{\operatorname{deg}_{\mathfrak{5}}(u) \cdot \operatorname{deg}_{\mathfrak{5}}(v)}{\operatorname{deg}_{\mathfrak{5}}(u)+\operatorname{deg}_{\mathfrak{5}}(v)-2}\right]^{3}}\right]$ $=\log (A(\mathfrak{b}))-\frac{1}{A(\mathfrak{b})} \log \left[\left[[8(f k+k t+f t)+4(f+k+t)] \times\left[\left(\frac{9}{4}\right)^{3}\right]^{\left(\frac{9}{4}\right)^{3}}\right] \times[8(f k+\right.$ $\left.k t+f t)+8(f+k+t)-48] \times\left[\left(\frac{12}{5}\right)^{3}\right]^{\left(\frac{12}{5}\right)^{3}}\right] \times[[48 k k t-20(f k+k t+f t)-$ $\left.\left.12(f+k+t)+48] \times\left[\left(\frac{8}{3}\right)^{3}\right]^{\left(\frac{8}{3}\right)^{3}}\right]\right]$

Where $A(\mathfrak{b})=\frac{1}{54000}(49152 f k t-9587282(f k+f t+k t)-3855657(f+k+t))-$ $\frac{1285219}{18000} t+\frac{277504}{1125}$

\section{First redefined Zagreb entropy}

$$
\begin{aligned}
& \operatorname{ReZG}_{1}(\Gamma)=(4 / 3) f+(4 / 3) k+(4 / 3) t-4+24 f k t
\end{aligned}
$$

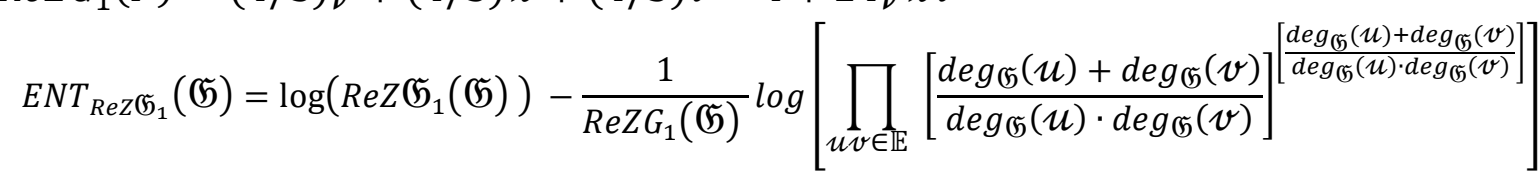

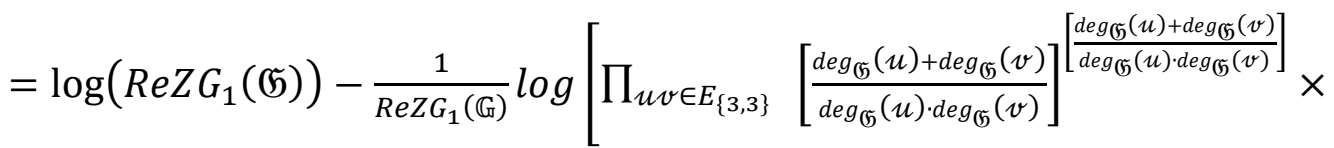

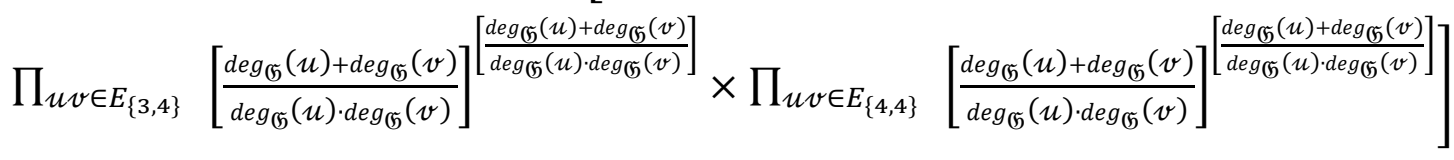




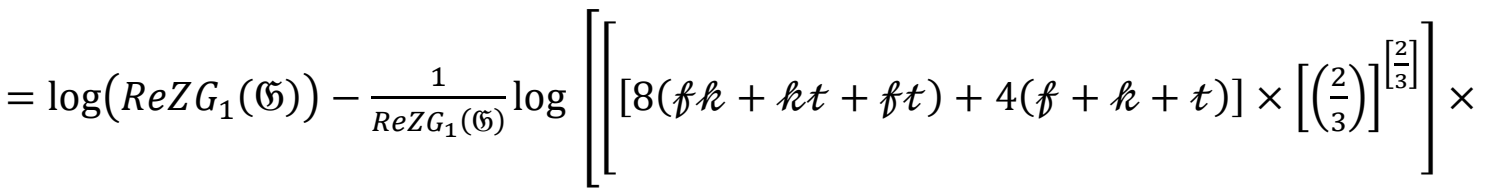

$$
\begin{aligned}
& {\left[[8(f k+k t+f t)+8(f+k+t)-48] \times\left[\left(\frac{7}{12}\right)\right]^{\left[\frac{7}{12}\right]}\right] \times[[48 f k t-20(f k+k t+} \\
& \left.\left.f t)-12(f+k+t)+48] \times\left[\left(\frac{1}{2}\right)\right]^{\left[\frac{1}{2}\right]}\right]\right]
\end{aligned}
$$

Where $\operatorname{ReZG}_{1}(\mathfrak{5})=(4 / 3) f+(4 / 3) k+(4 / 3) t-4+24 f k t$

\section{Second redefined Zagreb entropy}

$$
\begin{aligned}
& R e Z G_{2}(5)=(1 / 7)((672 t-100) k-100 t-30) f+(1 / 7)(-100 t-30) k-(30 / \\
& \text { 7) } t+96 / 7 \\
& E N T_{R e Z G_{2}}(\mathfrak{5})=\log \left(\operatorname{ReZG} G_{2}(\mathfrak{5})\right)-\frac{1}{R e Z G_{2}(\mathfrak{F})} \log \left[\prod_{u v \in \mathbb{E}}\left[\frac{\operatorname{deg}_{\mathfrak{5}}(u) \cdot \operatorname{deg}_{\mathfrak{5}}(v)}{\operatorname{deg}_{\mathfrak{5}}(u)+\operatorname{deg}_{\mathfrak{5}}(v)}\right]^{\left[\frac{\operatorname{deg}_{\mathfrak{5}}(u) \cdot \operatorname{deg}_{\mathfrak{5}}(v)}{\operatorname{deg}_{\mathfrak{5}}(u)+\operatorname{leg}_{\mathfrak{F}}(v)}\right]}\right] \\
& =\log \left(R e Z G_{2}(\mathfrak{5})\right)-\frac{1}{R e Z G_{2}(\mathfrak{G})} \log \left[\prod_{u v \in E_{\{3,3\}}}\left[\frac{\operatorname{deg}_{\mathfrak{F}}(u) \cdot \operatorname{deg}_{\mathfrak{5}}(v)}{\operatorname{deg}_{\mathfrak{5}}(u)+\operatorname{deg}_{\mathfrak{F}}(v)}\right]^{\left[\frac{\operatorname{deg}_{\mathfrak{5}}(u) \cdot \operatorname{deg} g_{\mathfrak{F}}(v)}{\operatorname{deg}_{\mathfrak{F}}(u)+\operatorname{deg}_{\mathfrak{F}}(v)}\right]} \times\right. \\
& \left.\prod_{u v \in E_{\{3,4\}}}\left[\frac{\operatorname{deg}_{\mathfrak{5}}(u) \cdot \operatorname{deg}_{\mathfrak{5}}(v)}{\operatorname{deg}_{\mathfrak{5}}(u)+\operatorname{deg}_{\mathfrak{5}}(v)}\right]^{\left[\frac{\operatorname{deg}_{\mathfrak{5}}(u) \cdot \operatorname{deg}_{\mathfrak{5}}(v)}{\operatorname{deg}_{\mathfrak{5}}(u)+\operatorname{deg}_{\mathfrak{5}}(v)}\right]} \times \prod_{u v \in E_{\{4,4\}}}\left[\frac{\operatorname{deg}_{\mathfrak{5}}(u) \cdot \operatorname{deg}_{\mathfrak{5}}(v)}{\operatorname{deg}_{\mathfrak{5}}(u)+\operatorname{deg}_{\mathfrak{5}}(v)}\right]^{\left[\frac{\operatorname{deg}_{\mathfrak{5}}(u) \cdot \operatorname{deg}_{\mathfrak{5}}(v)}{\operatorname{deg}_{\mathfrak{5}}(u)+\operatorname{deg}_{\mathfrak{5}}(v)}\right]}\right]
\end{aligned}
$$

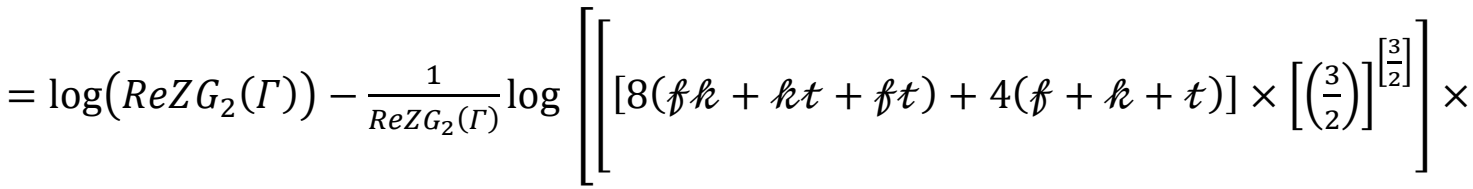

$$
\begin{aligned}
& {\left[[8(f k+k t+f t)+8(f+k+t)-48] \times\left[\left(\frac{12}{7}\right)\right]^{\left[\frac{12}{7}\right]}\right] \times[[48 k k t-20(f k+k t+} \\
& \left.f t)-12(f+k+t)+48] \times[2]^{[2]}\right]
\end{aligned}
$$

Where $\quad R e Z G_{2}(5)=(1 / 7)((672 t-100) k-100 t-30) f+(1 / 7)(-100 t-30) k-$ $(30 / 7) t+96 / 7$

\section{Third redefined Zagreb entropy}

$R e Z G_{3}(\mathfrak{5})=((6144 t-1456) k-1456 t-648) f+(-1456 t-648) k-648 t+$ 2112

$$
\begin{aligned}
& \operatorname{ENT}_{R e Z G_{3}}(\mathfrak{5})=\log \left(\operatorname{ReZG}_{3}(\mathfrak{5})\right)-\frac{1}{\operatorname{ReZG}_{3}(\mathfrak{5})} \log \left[\prod _ { u v \in E ( \Gamma ) } \left[( \operatorname { d e g } _ { \mathfrak { 5 } } ( u ) \cdot \operatorname { d e g } _ { \mathfrak { 5 } } ( v ) ) \cdot \left(\operatorname{deg}_{\mathfrak{5}}(u)+\right.\right.\right. \\
& \left.\left.\left.\operatorname{deg}_{\mathfrak{5}}(v)\right)\right]^{\left[\left(\operatorname{deg}_{\mathfrak{5}}(u) \cdot \operatorname{deg}_{\mathfrak{5}}(v)\right) \cdot\left(\operatorname{deg}_{\mathfrak{5}}(u)+\operatorname{deg}_{\mathfrak{5}}(v)\right)\right]}\right]
\end{aligned}
$$




$$
\begin{aligned}
& =\log \left(R e Z G_{3}(\mathfrak{5})\right)-\frac{1}{R e Z G_{3}(\mathfrak{G})} \log \left[\prod _ { u v \in E _ { \{ 3 , 3 \} } } \left[( \operatorname { d e g } _ { \mathfrak { 5 } } ( u ) \cdot \operatorname { d e g } _ { \mathfrak { 5 } } ( v ) ) \cdot \left(\operatorname{deg}_{\mathfrak{5}}(u)+\right.\right.\right. \\
& \left.\left.\operatorname{deg}_{\mathfrak{n}}(v)\right)\right] \times \prod_{w \in E_{\{3,4\}}}\left[\left(\operatorname{deg}_{\mathfrak{5}}(u) \cdot \operatorname{deg}_{\mathfrak{5}}(v)\right) \cdot\left(\operatorname{deg}_{\mathfrak{5}}(u)+\operatorname{deg}_{\mathfrak{5}}(v)\right)\right] \times \\
& \left.\prod_{u \in E_{\{4,4\}}}\left[\left(\operatorname{deg}_{\mathfrak{5}}(u) \cdot \operatorname{deg}_{\mathfrak{5}}(v)\right) \cdot\left(\operatorname{deg}_{\mathfrak{5}}(u)+\operatorname{deg}_{\mathfrak{5}}(v)\right)\right]\right] \\
& =\log \left(R e Z G_{3}(\mathfrak{5})\right)-\frac{1}{R e Z G_{3}(\Gamma)} \log \left[\left[[8(f k+k t+f t)+4(f+k+t)] \times[54]^{[54]}\right] \times\right. \\
& {\left[[8(f k+k t+f t)+8(f+k+t)-48] \times[84]^{[84]}\right] \times[[48 f k t-20(f k+k t+f t)-} \\
& \left.\left.12(f+k+t)+48] \times[128]^{[128]}\right]\right]
\end{aligned}
$$

Where $\quad R e Z G_{3}(5)=((6144 t-1456) k-1456 t-648) f+(-1456 t-648) k-$ $648 t+2112$

\section{Atom bond connectivity entropy}

$$
\begin{aligned}
& A B C(\mathfrak{5})=(1 / 3)(((36 t-15) k-15 t-9) f+(-15 t-9) k-9 t+36) \sqrt{2}+4((k+ \\
& t+1) f+(t+1) k+t-6) \sqrt{5}) \sqrt{3}+(1 / 3)(16 k+16 t+8) f+(1 / 3)(16 t+8) k+ \\
& (8 / 3) t \\
& E N T_{A B C}(\mathfrak{5})=\log (A B C((5))- \\
& \frac{1}{A B C(\mathfrak{5})} \log \left[\prod_{w \in \mathbb{E}}\left[\sqrt{\frac{\operatorname{deg}_{\mathfrak{5}}(u)+\operatorname{deg}_{\mathfrak{5}}(v)-2}{\operatorname{deg}_{\mathfrak{5}}(u) \cdot \operatorname{deg}_{\mathfrak{5}}(v)}}\right]^{\left[\sqrt{\left.\frac{\operatorname{deg}_{\mathfrak{5}(u)+\operatorname{deg}_{\mathfrak{5}}(v)-2}}{\operatorname{deg}_{\mathfrak{5}}(u) \cdot \operatorname{deg}_{\mathfrak{5}}(v)}\right]}\right]}\right.
\end{aligned}
$$

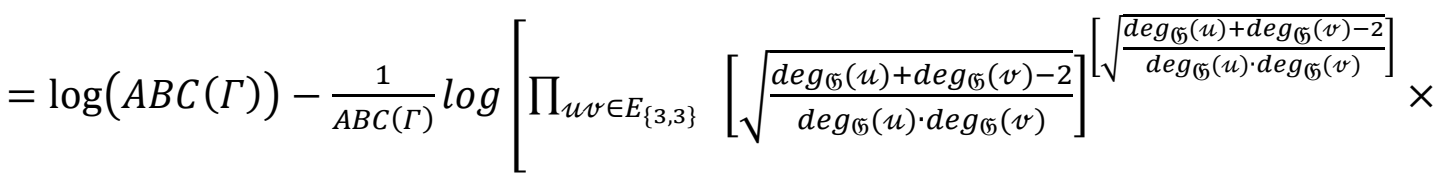$$
\prod_{w \in E_{\{3,4\}}}\left[\sqrt{\frac{\operatorname{deg}_{\mathfrak{5}}(u)+\operatorname{deg}_{\mathfrak{5}}(v)-2}{\operatorname{deg}_{\mathfrak{5}}(u) \cdot \operatorname{deg}_{\mathfrak{5}}(v)}}\right]^{\left[\sqrt{\frac{\operatorname{deg}_{\mathfrak{G}}(u)+\operatorname{deg}_{\mathfrak{5}}(v)-2}{\operatorname{leg}_{\mathfrak{5}}(u) \cdot \operatorname{deg}_{\mathfrak{G}}(v)}}\right]} \times
$$$$
\left.\prod_{u v \in E_{\{4,4\}}}\left[\sqrt{\frac{\operatorname{deg}_{\mathfrak{5}}(u)+\operatorname{deg}_{\mathfrak{5}}(v)-2}{\operatorname{deg}_{\mathfrak{5}}(u) \cdot \operatorname{deg}_{\mathfrak{F}}(v)}}\right]^{\left[\sqrt{\frac{\operatorname{deg}_{\mathfrak{5}}(u)+\operatorname{deg}_{\mathfrak{5}}(v)-2}{\operatorname{deg}_{\mathfrak{5}}(u) \cdot \operatorname{deg}_{\mathfrak{5}}(v)}}\right]}\right]
$$$$
=\log (A B C(\mathfrak{5}))-\frac{1}{A B C(\mathfrak{5})} \log \left[\left[[8(f k+k t+f t)+4(f+k+t)] \times\left[\left(\frac{2}{3}\right)\right]^{\left[\frac{2}{3}\right]}\right] \times\right.
$$$$
\left.\left[[8(f k+k t+f t)+8(f+k+t)-48] \times\left[\left(\sqrt{\frac{5}{12}}\right)\right]^{\left[\sqrt{\frac{5}{12}}\right.}\right]\right] \times[[48 k t-20(f k+k t+
$$$$
\left.\left.f t)-12(f+k+t)+48] \times\left[\left(\sqrt{\frac{3}{8}}\right)\right]^{\left[\sqrt{\frac{3}{8}}\right]}\right]\right]
$$

Where $A B C(\mathfrak{5})=(1 / 3)((((36 t-15) k-15 t-9) f+(-15 t-9) k-9 t+36) \sqrt{2}+$ $4((k+t+1) f+(t+1) k+t-6) \sqrt{5}) \sqrt{3}+(1 / 3)(16 k+16 t+8) f+(1 / 3)(16 t+$ 8) $k+(8 / 3) t$ 


\section{Geometric arithmetic entropy}

$G A(\mathfrak{5})=(1 / 7)((32 k+32 t+32) f+(32 t+32) k+32 t-192)) \sqrt{3}+(1 /$

7) $((336 t-84) k-84 t-56) f+(1 / 7)(-84 t-56)) k-8 t+48$

$E N T_{G A}(\mathfrak{5})=\log (G A(\mathfrak{5}))-\frac{1}{G A(\mathfrak{G})} \log \left[\prod_{w \in \mathbb{E}}\left[\frac{2 \sqrt{\operatorname{deg}_{\mathfrak{5}}(u) \cdot \operatorname{deg}_{\mathfrak{5}}(v)}}{\operatorname{deg}_{\mathfrak{5}}(u)+\operatorname{deg}_{\mathfrak{5}}(v)}\right]^{\left[\frac{2 \sqrt{\operatorname{deg}_{\mathfrak{5}}(u) \cdot \operatorname{deg}_{\mathfrak{5}}(v)}}{\operatorname{leg}_{\mathfrak{5}}(u)+\operatorname{deg}_{\mathfrak{5}}(v)}\right]}\right]$

$=\log (G A(\mathfrak{5}))-\frac{1}{G A(\mathfrak{F})} \log \left[\prod_{w \in E_{\{3,3\}}}\left[\frac{2 \sqrt{\operatorname{deg}_{\mathfrak{5}}(u) \cdot \operatorname{deg}_{\mathfrak{5}}(v)}}{\operatorname{deg}_{\mathfrak{G}}(u)+\operatorname{deg}_{\mathfrak{G}}(v)}\right]^{\left[\frac{2 \sqrt{\operatorname{deg}_{\mathfrak{5}}(u) \cdot \operatorname{deg}_{\mathfrak{5}}(v)}}{\operatorname{leg}_{\mathfrak{F}}(u)+\operatorname{deg}_{\mathfrak{F}}(v)}\right.}\right] \times$

$\prod_{u v \in E_{\{3,4\}}}\left[\frac{2 \sqrt{\operatorname{deg}_{\mathfrak{5}}(u) \cdot \operatorname{deg}_{\mathfrak{5}}(v)}}{\operatorname{deg}_{\mathfrak{5}}(u)+\operatorname{deg}_{\mathfrak{5}}(v)}\right]^{\left[\frac{2 \sqrt{\operatorname{deg}_{\mathfrak{5}}(u) \cdot \operatorname{deg}_{\mathfrak{5}}(v)}}{\operatorname{deg}_{\mathfrak{5}}(u)+\operatorname{deg}_{\mathfrak{5}}(v)}\right]} \times$

$\left.\prod_{w v \in E_{\{4,4\}}}\left[\frac{2 \sqrt{\operatorname{deg}_{\mathfrak{F}}(u) \cdot \operatorname{deg}_{\mathfrak{F}}(v)}}{\operatorname{deg}_{\mathfrak{F}}(u)+\operatorname{deg}_{\mathfrak{F}}(v)}\right]^{\left[\frac{2 \sqrt{\operatorname{deg}_{\mathfrak{G}}(u) \cdot \operatorname{deg}_{\mathfrak{G}}(v)}}{\operatorname{deg}_{\mathfrak{G}}(u)+\operatorname{deg}_{\mathfrak{F}}(v)}\right]}\right]$

$=\log \left(G A((5))-\frac{1}{G A(\mathfrak{G})} \log \left[\left[[8(f k+k t+f t)+4(f+k+t)] \times[1]^{[1]}\right] \times[[8(f k+\right.\right.$

$\left.k t+f t)+8(f+k+t)-48] \times\left[\left(\frac{4 \sqrt{3}}{7}\right)\right]^{\left[\frac{4 \sqrt{3}}{7}\right]}\right] \times[[48 k k t-20(f k+k t+f t)-$

$\left.\left.12(f+k+t)+48] \times[1]^{[1]}\right]\right]$

Where $\quad G A(\mathfrak{5})=(1 / 7)((32 k+32 t+32) f+(32 t+32) k+32 t-192)) \sqrt{3}+(1 /$ 7) $((336 t-84) k-84 t-56) f+(1 / 7)(-84 t-56)) k-8 t+48$

\section{Forgotten entropy}

$F(\mathfrak{b})=1536 f k t-296(f k+f t+k t)-112(f+k+t)+336$

$\operatorname{ENT}_{F}(\mathfrak{5})=\log (F(\mathfrak{5}))-\frac{1}{F(\mathfrak{F})} \log \left[\prod_{w \in \mathbb{E}}\left[\operatorname{deg}_{\mathfrak{5}}(u)^{2}+\operatorname{deg}_{\mathfrak{5}}(v)^{2}\right]^{\left[\operatorname{deg}_{\mathfrak{5}}(u)^{2}+\operatorname{deg}_{\mathfrak{5}}(v)^{2}\right]}\right]$

$=\log (F(\mathfrak{5}))-\frac{1}{F(\mathfrak{5})} \log \left[\prod_{\mathfrak{w} \in E_{\{3,3\}}}\left[\operatorname{deg}_{\mathfrak{5}}(u)^{2}+\operatorname{deg}_{\mathfrak{5}}(v)^{2}\right]^{\left[\operatorname{deg}_{\mathfrak{5}}(u)^{2}+\operatorname{deg}_{\mathfrak{F}}(v)^{2}\right]} \times\right.$

$\prod_{u v \in E_{\{3,4\}}}\left[\operatorname{deg}_{\mathfrak{5}}(u)^{2}+\operatorname{deg}_{\mathfrak{G}}(v)^{2}\right]^{\left[\operatorname{deg}_{\mathfrak{F}}(u)^{2}+\operatorname{deg}_{\mathfrak{F}}(v)^{2}\right]} \times \prod_{u v \in E_{\{4,4\}}}\left[\operatorname{deg}_{\mathfrak{5}}(u)^{2}+\right.$

$\left.\left.\operatorname{deg}_{\mathfrak{5}}(v)^{2}\right]^{\left[\operatorname{deg}_{\mathfrak{F}}(u)^{2}+\operatorname{deg}_{\mathfrak{5}}(v)^{2}\right]}\right]$

$=\log (F(\mathfrak{b}))-\frac{1}{F(\mathfrak{G})} \log \left[\left[[8(f k+k t+f t)+4(f+k+t)] \times[18]^{[18]}\right] \times[[8(f k+\right.$

$\left.k t+f t)+8(f+k+t)-48] \times[25]^{[25]}\right] \times[[48 f k t-20(f k+k t+f t)-12(f+$

$\left.\left.k+t)+48] \times[32]^{[32]}\right]\right]$

Where $F(5)=1536 f k t-296(f k+f t+k t)-112(f+k+t)+336$ 


\section{Sum-connectivity entropy}

$$
\begin{aligned}
& \chi((5)=(1 / 21)(((28 k+28 t+14) f+(28 t+14) k+14 t) \sqrt{3}+((252 t-105) k- \\
& 105 t-63) f+(-105 t-63) k-63 t+252) \sqrt{2}+(8 / 7)((k+t+1) f+(t+1) k+ \\
& t-6) \sqrt{7}
\end{aligned}
$$$$
\operatorname{ENT}_{\chi}(\mathfrak{5})=\log (\chi(\mathfrak{5}))-\frac{1}{\chi(\mathfrak{5})} \log \left[\prod_{w \in \mathbb{E}}\left[\frac{1}{\sqrt{\operatorname{deg}_{\mathfrak{F}}(u)+\operatorname{deg}_{\mathfrak{F}}(v)}}\right]^{\left[\frac{1}{\sqrt{\operatorname{leg}_{\mathfrak{G}}(u)+\operatorname{deg}_{\mathfrak{F}}(v)}}\right]}\right]
$$$$
=\log (\chi(\mathfrak{5}))-\frac{1}{F(\mathfrak{G})} \log \left[\prod_{u v \in E_{\{3,3\}}}\left[\frac{1}{\sqrt{\operatorname{deg}_{\mathfrak{5}}(u)+\operatorname{deg}_{\mathfrak{5}}(v)}}\right]^{\left[\frac{1}{\sqrt{\operatorname{leg}_{\mathfrak{G}}(u)+\operatorname{deg}_{\mathfrak{F}}(v)}}\right]} \times\right.
$$

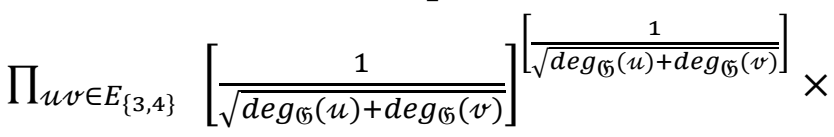$$
\left.\prod_{u v \in E_{\{4,4\}}}\left[\frac{1}{\sqrt{\operatorname{deg}_{\mathfrak{5}}(u)+\operatorname{deg}_{\mathfrak{5}}(v)}}\right]^{\left[\frac{1}{\sqrt{\operatorname{deg}_{\mathfrak{5}}(u)+\operatorname{deg}_{\mathfrak{5}(v)}}}\right]}\right]
$$$$
=\log (\chi(\mathfrak{b}))-\frac{1}{\chi(\mathfrak{5})} \log \left[\left[[8(f k+k t+f t)+4(f+k+t)] \times\left[\frac{1}{\sqrt{6}}\right]^{\left[\frac{1}{\sqrt{6}}\right]}\right] \times[[8(f k+\right.
$$$$
\left.k t+f t)+8(f+k+t)-48] \times\left[\frac{1}{\sqrt{7}}\right]^{\left[\frac{1}{\sqrt{7}}\right]}\right] \times[[48 k k t-20(f k+k t+f t)-12(f+
$$

$\left.\left.k+t)+48] \times\left[\frac{1}{\sqrt{8}}\right]^{\left[\frac{1}{\sqrt{8}}\right]}\right]\right]$

Where $\chi(5)=(1 / 21)(((28 k+28 t+14) f+(28 t+14) k+14 t) \sqrt{3}+((252 t-$ $105) k-105 t-63) f+(-105 t-63) k-63 t+252) \sqrt{2}+(8 / 7)((k+t+1) f+$ $(t+1) k+t-6) \sqrt{7}$

Theorem 2. If $\mathfrak{b}$ is a zeolite LTA framework, then the Irregularity-based molecular descriptors are computed as

1. $\operatorname{VAR}(55)=\frac{1}{36}\left(432 f k t^{2}+(-24 f-24 k-24 t-60 f k-60 f t-60 k t+\right.$ 72) $\left.f k t-(f k+f t+k t)^{2}\right) / f k t^{2}$

2. $I R 1(\mathfrak{5})=\frac{1}{3} f k t\left(2304 f k t^{2}+(-240 f-240 k-240 t-360 f k-360 f t-\right.$ $360 k t+720) f k t-(8((7 / 2) f k+(7 / 2) f t+(7 / 2) k t+f+k+t-$

3)) $(f k+f t+k t))$

3. $I R 2\left((5)=\sqrt{\left(\frac{768 k h t-152(f k+f t+h t)-60(f+k+t)+192}{48 f k t-4(f h+h t+f t)}\right)}-\frac{2(48 f k t-4(f k+k t+f t))}{24 f k t}\right.$

4. $\operatorname{IRDIF(5)}=2 f k+2 k t+2 f t+2 f+2 k+2 t-12$

5. $A L(\mathfrak{5})=8(f k+k t+f t)+8(f+k+t)-48$

6. $\operatorname{IRL}(\mathfrak{5})=0.2877(8(f k+k t+f t)+8(f+k+t)-48)$

7. $\operatorname{IRLU}(\mathfrak{5})=\frac{1}{3}(8(f k+k t+f t)+8(f+k+t)-48)$

8. $\operatorname{IRLF}(\mathfrak{5})=\frac{1}{\sqrt{12}}(8(f k+k t+f t)+8(f+k+t)-48)$ 
9. $\operatorname{IRF}(\mathfrak{5})=8 f k+8 f t+8 k t+8 f+8 k+8 t-48$

10. $\operatorname{IRLA}(\mathfrak{5})=\frac{2}{7}(8(f k+k t+f t)+8(f+k+t)-48)$

11. $\operatorname{IRFW}(\mathfrak{5})=\frac{-2 f k-2 f t-2 k t-2 f-2 k-2 t+12}{-192 f k t+38 f k+38 f t+38 k t+15 f+15 k+15 t-48}$

12. $\operatorname{IRA} A(\mathfrak{5})=0.0059856(8(f k+k t+f t)+8(f+k+t)-48)$

13. $\operatorname{IRB}((5)=0.07179676949(8(f k+k t+f t)+8(f+k+t)-48)$

14. $\operatorname{IRB}(\mathfrak{G})=$ $\frac{(16 f+16 f k+16 f t+16 k+16 k t+16 t-96) \sqrt{3}-44 f-44 k-44 t-56 f k-56 f t-56 k t+192 f k t+192}{-19}$ $48 f k t-4(f k+k t+f t)$ $\frac{48 f k t-4(f k+k t+f t)}{24 f k t}$

15. $I R G A(\mathfrak{G})=0.2798078943 \cdot(8(f k+k t+f t)+8(f+k+t)-48)$

16. $I R R_{t}(\mathfrak{5})=4 f k+4 f t+4 f t+4 f+4 k+4 t-24$

Proof: Let $(\mathfrak{G}=Z[f, k, t]$ be a Zeolite graph. The cardinality of $Z[f, k, t]$ is depicted as $n=$ $|V(\Gamma)|=24 f k t$ and $m=|E(\Gamma)|=48 f k t-4(f k+k t+f t)$. Using the definitions of Irregularity-based indices and the Table 3 , we get the following result as

1. $\operatorname{VAR}(55)=\frac{1}{36}\left(432 f k t^{2}+(-24 f-24 k-24 t-60 f k-60 f t-60 k t+\right.$ 72) fkt $\left.-(f k+f t+k t)^{2}\right) / f k t^{2}$

2. $I R 1(\mathfrak{5})=\frac{1}{3} f k t\left(2304 f k t^{2}+(-240 f-240 k-240 t-360 f k-360 f t-\right.$ $360 k t+720) f k t-(8((7 / 2) f k+(7 / 2) f t+(7 / 2) k t+f+k+t-$ 3)) $(f k+f t+k t))$

3. $I R 2\left((5)=\sqrt{\left(\frac{768 k h t-152(f k+f t+k t)-60(f+k+t)+192}{48 f k t-4(f k+k t+f t)}\right)}-\frac{2(48 f k t-4(f k+k t+f t))}{24 f k t}\right.$

4. $\operatorname{IRDIF}(\mathfrak{5})=2 k k+2 k t+2 f t+2 k+2 k+2 t-12$

5. $A L(5)=8(f k+k t+f t)+8(f+k+t)-48$

6. $\operatorname{IRL}(\mathfrak{G})=0.2877(8(f k+k t+f t)+8(f+k+t)-48)$

7. $\operatorname{IRLU}(\mathfrak{5})=\frac{1}{3}(8(f k+k t+f t)+8(f+k+t)-48)$

8. $\operatorname{IRLF}(\mathfrak{5})=\frac{1}{\sqrt{12}}(8(f k+k t+f t)+8(f+k+t)-48)$

9. $\operatorname{IRF}(\mathfrak{5})=8 k k+8 f t+8 k t+8 f+8 k+8 t-48$

10. $\operatorname{IRLA}(\mathfrak{5})=\frac{2}{7}(8(f k+k t+f t)+8(f+k+t)-48)$

11. $I R F W(5)=\frac{-2 f k-2 f t-2 k t-2 f-2 k-2 t+12}{-192 k k t+38 f k+38 f t+38 k t+15 f+15 k+15 t-48}$

12. $\operatorname{IRA}(\mathfrak{G})=0.0059856(8(f k+k t+f t)+8(f+k+t)-48)$

13. $\operatorname{IRB}(\mathfrak{5})=0.07179676949(8(f k+k t+f t)+8(f+k+t)-48)$

14. $\operatorname{IRB}(\mathfrak{G})=$

$(16 f+16 f k+16 f t+16 k+16 k t+16 t-96) \sqrt{3}-44 f-44 k-44 t-56 f k-56 f t-56 k t+192 f k t+192$ $48 f k t-4(f k+k t+f t)$

$\frac{48 f k t-4(f k+k t+f t)}{24 f k t}$

15. $I R G A(\mathfrak{5})=0.2798078943 \cdot(8(f k+k t+f t)+8(f+k+t)-48)$

16. $I R R_{t}(\mathfrak{5})=4 f k+4 f t+4 f t+4 f+4 k+4 t-24$

Hence the proof. 


\section{Numerical Analysis}

In this section, the numerical results obtained from the expressions of the VDB indices for the zeolite LTA framework are presented. The zeolite LTA framework is represented as $Z[f, k, t]$. The values of the VDB indices for this system for different values of $f, k$, and $t$ are presented in tables 4 and 5 for numerical comparison. These values have been plotted using the Origin 2020b software for a graphical comparison of the computed topological descriptors (see Figures 3 and 4). These graphs provide a visual representation of the values of different indices with respect to the varying topology of the zeolite framework.

Table 4. Numerical calculations of Theorem 2.

\begin{tabular}{c|c|c|c|c|c|c}
{$[\boldsymbol{f}, \boldsymbol{k}, \boldsymbol{t}]$} & $\mathbf{M}_{\mathbf{2}}{ }^{\mathbf{m}}(\mathbf{G})$ & $\mathbf{R e Z G}_{\mathbf{1}}(\mathbf{G})$ & $\mathbf{R e Z G}_{\mathbf{2}}(\mathbf{G})$ & $\mathbf{R e Z G}_{\mathbf{3}}(\mathbf{G})$ & $\mathbf{S K}(\mathbf{G})$ & $\mathbf{S K}_{\mathbf{1}}(\mathbf{G})$ \\
\hline$[1,1,1]$ & 4.0 & 24.0 & 54.0 & 1944.0 & 108.0 & 162.0 \\
\hline$[2,2,2]$ & 28.8 & 196.0 & 584.6 & 29904.0 & 1176.0 & 2076.0 \\
\hline$[3,3,3]$ & 91.5 & 656.0 & 2181.4 & 122856.0 & 4380.0 & 8142.0 \\
\hline$[4,4,4]$ & 210.0 & 1548.0 & 5420.6 & 317664.0 & 10872.0 & 20664.0 \\
\hline$[5,5,5]$ & 402.3 & 3016.0 & 10878.0 & 651192.0 & 21804.0 & 41946.0 \\
\hline$[6,6,6]$ & 686.5 & 5204.0 & 19129.7 & 1160304.0 & 38328.0 & 74292.0 \\
\hline$[7,7,7]$ & 1080.5 & 8256.0 & 30751.7 & 1881864.0 & 61596.0 & 120006.0 \\
\hline$[8,8,8]$ & 1602.3 & 12316.0 & 46320.0 & 2852736.0 & 92760.0 & 181392.0 \\
\hline$[9,9,9]$ & 2270.0 & 17528.0 & 66410.6 & 4109784.0 & 132972.0 & 260754.0
\end{tabular}
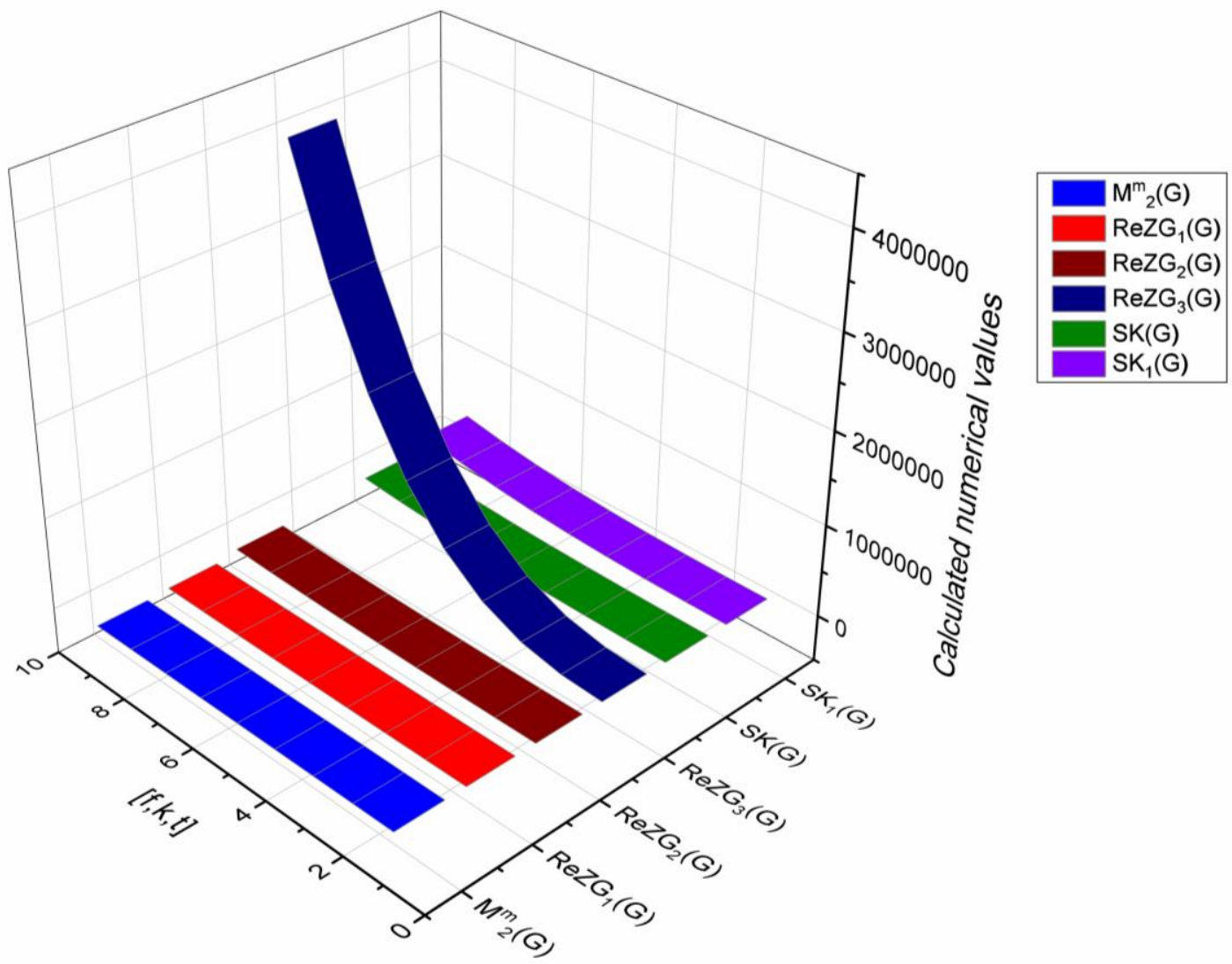

Figure 3. 3D Graphical representation of Table 4.

Table 5. Numerical calculations of Theorem 3.

\begin{tabular}{c|c|c|c|c|c}
{$[\boldsymbol{f}, \boldsymbol{k}, \boldsymbol{t}]$} & $\mathbf{S K}_{\mathbf{2}}(\mathbf{G})$ & $\mathbf{A G}_{\mathbf{1}}(\mathbf{G})$ & $\mathbf{F}(\mathbf{G})$ & $\boldsymbol{\chi}(\mathbf{G})$ & $\mathbf{S D D}(\mathbf{G})$ \\
\hline$[1,1,1]$ & 324.0 & 36.0 & 648.0 & 14.7 & 72.0 \\
\hline$[2,2,2]$ & 4176.0 & 337.0 & 8400.0 & 127.7 & 680.0 \\
\hline$[3,3,3]$ & 16344.0 & 1190.5 & 32808.0 & 439.7 & 2396.0 \\
\hline$[4,4,4]$ & 41436.0 & 2884.5 & 83088.0 & 1052.4 & 5796.0
\end{tabular}




\begin{tabular}{c|c|c|c|c|c}
{$[\boldsymbol{f}, \boldsymbol{k}, \boldsymbol{t}]$} & $\mathbf{S K}_{\mathbf{2}}(\mathbf{G})$ & $\mathbf{A G}_{\mathbf{1}}(\mathbf{G})$ & $\mathbf{F}(\mathbf{G})$ & $\boldsymbol{\chi}(\mathbf{G})$ & $\mathbf{S D D}(\mathbf{G})$ \\
\hline$[5,5,5]$ & 84060.0 & 5707.0 & 168456.0 & 2067.8 & 11456.0 \\
\hline$[6,6,6]$ & 148824.0 & 9945.9 & 298128.0 & 3587.5 & 19952.0 \\
\hline$[7,7,7]$ & 240336.0 & 15889.4 & 481320.0 & 5713.6 & 31860.0 \\
\hline$[8,8,8]$ & 363204.0 & 23825.4 & 727248.0 & 8547.7 & 47756.0 \\
\hline$[9,9,9]$ & 522036.0 & 34041.9 & 1045128.0 & 12191.7 & 68216.0
\end{tabular}

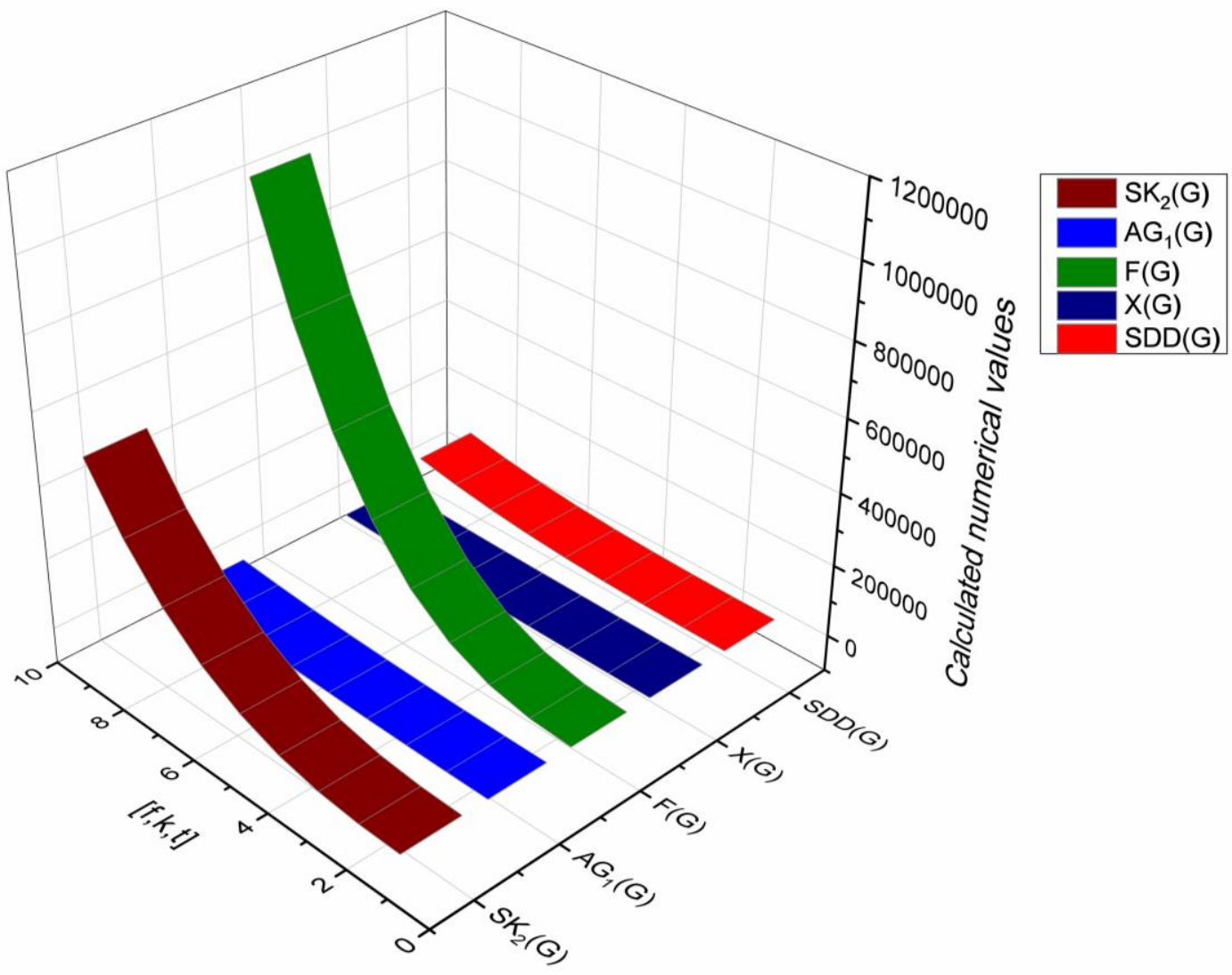

Figure 4. 3D Graphical representation of Table 5.

\section{Conclusions}

The computed analytical expressions of VDB indices, VDB entropy measures, and VDB Irregularity indices, in turn, serve as tools to forecast several properties of molecular compounds without laboratory experiments. In this context, the VDB indices play a crucial role in characterizing the molecular structure into a real number in order to predict the important properties of chemical compounds. Therefore, we believe that our results could play an important role in predicting the different properties of zeolite systems. We have also corroborated our computed results with graphical representations of the topological indices. This study could be used as a guide for future researchers to develop new zeolites.

\section{Funding}

This research received no external funding.

\section{Acknowledgment}

The authors would like to express their gratitude to the management of Sri Sivasubramaniya Nadar College of Engineering, Kalavakkam-603110, for their unwavering support and encouragement in carrying out this research. 


\section{Conflict of interest}

The authors state that the publishing of this paper does not include any conflicts of interest.

\section{References}

1. Jiri, C.; Herman, V.B.; Avelino, C.; Ferdi, S. Introduction to zeolite science and practice. Elsevier, Studies in Surface Science 2007, 2, 1058-1065.

2. Szostak, R. Molecular Sieves: Principles of Synthesis and Identification. Blackie Academic \& Professional, London 1998.

3. Earl, D.J.; Deem, M.W. Toward a database of hypothetical zeolite structures. Industrial \& engineering chemistry research 2006, 45, 5449-5454.

4. West, D.B. Introduction to graph theory. Upper Saddle River, NJ: Prentice Hall 1996, 2.

5. Julietraja, K.; Venugopal, P. Computation of Degree-Based Topological Descriptors Using M-Polynomial for Coronoid Systems. Polycyclic Aromatic Compounds (advance online publication) 2020, https://doi.org/10.1080/10406638.2020.1804415.

6. Julietraja, K.; Venugopal, P.; Prabhu, S.; Liu, J.B. M-polynomial and degree-based Molecular Descriptors of Certain Classes of Benzenoid Systems. Polycyclic Aromatic Compounds (Accepted for Publication) 2020, https://doi.org/10.1080/10406638.2020.1867205.

7. Julietraja, K.; Venugopal, P.; Prabhu, S.; Deepa, S.; Siddiqui, M.K. Molecular Structural Descriptors of Donut Benzenoid Systems. Polycyclic Aromatic Compounds (advance online publication) 2021, https://doi.org/10.1080/10406638.2021.1885456.

8. Julietraja, K.; Venugopal, P.; Prabhu, S.; Arulmozhi, A.; Siddiqui, M.K. Structural Analysis of Three Types of PAHs using Entropy Measures. Polycyclic Aromatic Compounds (advance online publication) 2021, https://doi.org/10.1080/10406638.2021.1884101.

9. Mondal, S.; Siddiqui, M.K.; De, N.; Pal, A. Neighborhood M-polynomial of crystallographic structures. Biointerface research in applied chemistry 2021, 11, 9372-9381, https://doi.org/10.33263/BRIAC112.93729381.

10. Mondal, S.; De, N.; Siddiqui, M.K.; Pal, A. Topological Properties of Para-Line Graph of Some Convex Polytopes Using Neighborhood M-Polynomial. Biointerface research in applied chemistry 2020, 11, 9915 9927, https://doi.org/10.33263/BRIAC113.99159927.

11. Mondal, S.; De, N.; Pal, A. Multiplicative degree based topological indices of nanostar dendrimers. Biointerface research in applied chemistry 2021, 11, 7700-7711.

12. Julietraja, K.; Venugopal, P.; Chellamani, P. Topological Analysis of PAHs using Irregularity based Indices. Biointerface research in applied chemistry 2021, 12, 2970-2987 https://doi.org/10.33263/BRIAC123.29702987

13. Nilanjan, De. Coputation of General Zagreb Index of Nanotubes coverd by $\mathrm{C}_{5}$ and $\mathrm{C}_{7}$. Biointerface research in applied chemistry 2021, 11, 8001-8008.

14. Ravi, V.; Desikan, K. Neighbourhood Degree-Based Topological Indices of Graphene Structure. Biointerface Research in Applied Chemistry 2021, 11, 13681-13694.

15. Afzal, F.; Hussain, S.; Afzal, D.; Razaq, S. Some new degree based topological indices via Mpolynomial. Journal of Information and Optimization Sciences 2020, 41, 1061-1076.

16. Raza, Z.; Sukaiti, E.K. M-polynomial and degree based topological indices of some nanostructures. Symmetry 2020, $12,831$.

17. De, N.; Cancan, M.; Alaeiyan, M.; Farahani, M.R. On some degree based topological indices of mkgraph. Journal of Discrete Mathematical Sciences and Cryptography 2020, 23, 1183-1194.

18. Monsalve, J.; Rada, J.; Vertex-degree based topological indices of digraphs. Discrete Applied Mathematics 2021, 295, 13-24.

19. Cruz, R.; Santamaría-Galvis, A.D.; Rada, J. Extremal values of vertex-degree-based topological indices of coronoid systems. International Journal of Quantum Chemistry 2021, 121, 26536.

20. Cancan, M.; Mondal, S.; De, N.; Pal, A.. Multiplicative degree based topological indices of some chemical structures in drug. Proyecciones (Antofagasta) 2020, 39, 1347-1364.

21. Ali, P.; Kirmani, S.A.K.; Al Rugaie, O.; Azam, F. Degree-based topological indices and polynomials of hyaluronic acid-curcumin conjugates. Saudi Pharmaceutical Journal 2020, 28, 1093-1100.

22. Shanmukha, M.C.; Basavarajappa, N.S.; Shilpa, K.C.; Usha, A. Degree-based topological indices on anticancer drugs with QSPR analysis. Heliyon 2020, 6, 04235. 
23. Afzal, D.; Afzal, F.; Farahani, M.R.; Ali, S. On Computation of Recently Defined Degree-Based Topological Indices of Some Families of Convex Polytopes via M-Polynomial. Complexity 2021.

24. Filipovski, S. Relations between Sombor Index and some Degree-Based Topological Indices. Iranian Journal of Mathematical Chemistry 2021, 12, 19-26.

25. Rafiullah, M.; Siddiqui, H.M.A.; Siddiqui, M.K.; Dhlamini, M. On Degree-Based Topological Indices for Strong Double Graphs. Journal of Chemistry 2021.

26. Prabhu, S.; Murugan, G.; Michael Cary, Arulperumjothi, M.; and Liu, J.B. On certain distance and degree based topological indices of Zeolite LTA frameworks. Materials Research Express, 2020, 7, 055006.

27. Wiener, H. Structural determination of paraffin boiling points. Journal of the American chemical society 1947, 69, 17-20.

28. Miličević, A.; Nikolić, S.; Trinajstić, N. On reformulated Zagreb indices. Molecular diversity 2004, 8, 393399.

29. Usha, A.; Ranjini, P.S.; Lokesha, V. Zagreb co-indices, augmented zagreb index, redefined zagreb indices and their polynomials for phenylene and hexagonal squeeze. In Proceedings of International Congress in Honour of Dr. Ravi. P. Agarwal, Uludag University, Bursa, Turkey 2014.

30. Ranjini, P.S.; Lokesha, V.; Usha, A. Relation between phenylene and hexagonal squeeze using harmonic index. Int. J. Graph Theory 2013, 1, 116-121.

31. Shegehalli, V.S.; Kanabur, R. Arithmetic-Geometric indices of some class of Graph. Journal of Computer and Mathematical sciences 2015, 6, 194-199.

32. Shigehalli, V.S.; Kanabur, R. Computation of new degree-based topological indices of graphene. Journal of Mathematics 2016.

33. Furtula, B.; Gutman, I. A forgotten topological index. Journal of Mathematical Chemistry 2015, 53, 11841190.

34. Gupta, C.K.; Lokesha, V.; Shwetha, S.B.; Ranjini, P.S. On the Symmetric Division deg Index of Graph. Southeast Asian Bulletin of Mathematics 2016, 40.

35. Sedlar, J.; Stevanović, D.; Vasilyev, A. On the inverse sum indeg index. Discrete Applied Mathematics 2015, 184, 202-212.

36. Zhou, B.; Trinajstić, N. On a novel connectivity index. Journal of mathematical chemistry 2009, 46, 12521270.

37. Shannon, C.E. A mathematical theory of communication. The Bell system technical journal 1948, 27, 379423.

38. Chen, Z.; Dehmer, M.; Shi, Y. A note on distance-based graph entropies. Entropy 2014, 16, 5416-27.

39. Chartrand, G.; Erdös, P.; Oellermann, O.R. How to define an irregular graph?. The College Mathematics Journal 1988, 19, 36-42.

40. Réti, T.; Sharafdini, R.; Dregelyi-Kiss, A.; Haghbin, H. Graph irregularity indices used as molecular descriptors in QSPR studies. MATCH Communications in Mathematical and in Computer Chemistry 2018, $79,509-524$. 